Provided for non-commercial research and education use. Not for reproduction, distribution or commercial use.

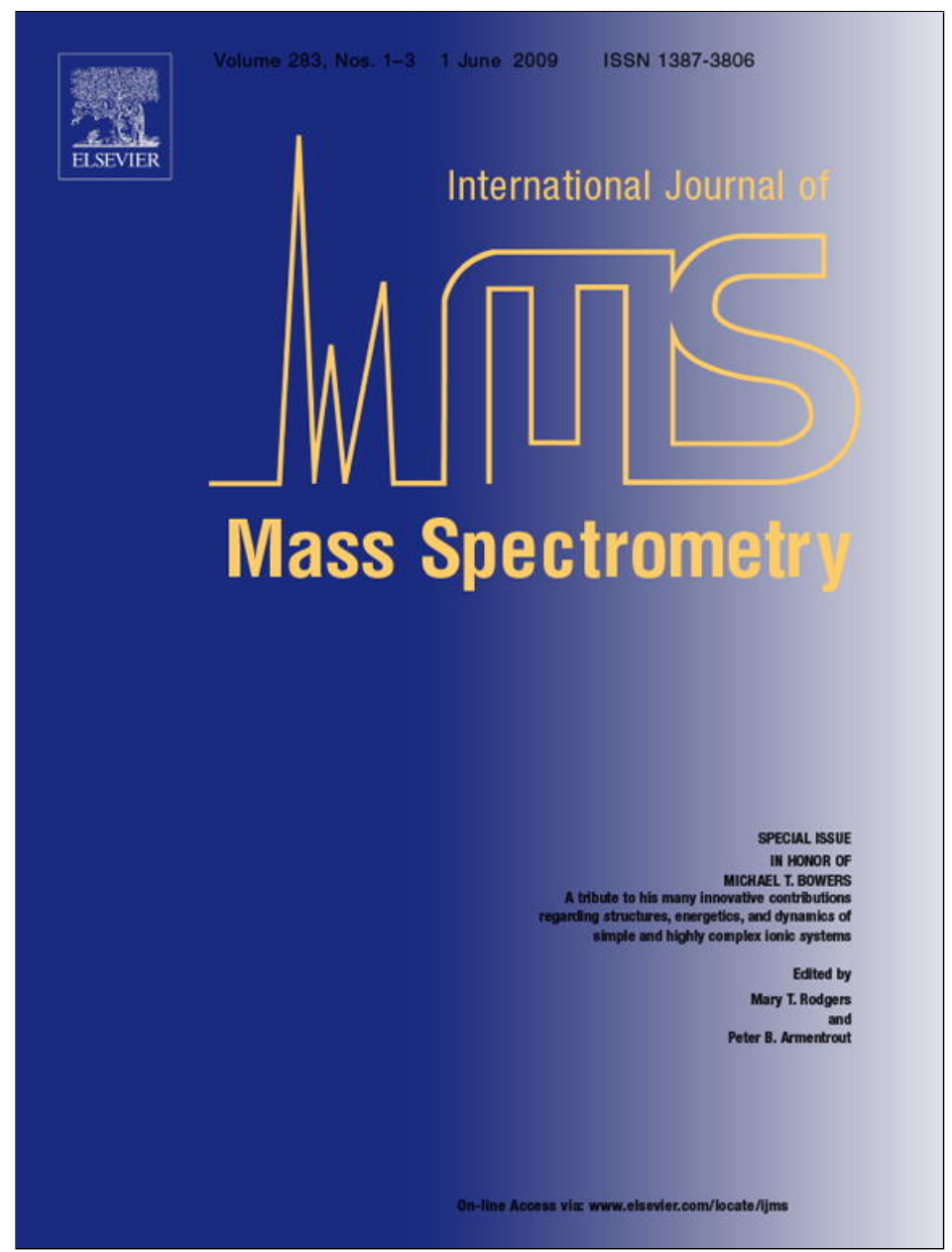

This article appeared in a journal published by Elsevier. The attached copy is furnished to the author for internal non-commercial research and education use, including for instruction at the authors institution and sharing with colleagues.

Other uses, including reproduction and distribution, or selling or licensing copies, or posting to personal, institutional or third party websites are prohibited.

In most cases authors are permitted to post their version of the article (e.g. in Word or Tex form) to their personal website or institutional repository. Authors requiring further information regarding Elsevier's archiving and manuscript policies are encouraged to visit:

http://www.elsevier.com/copyright 


\title{
Electron shuttling in electron transfer dissociation
}

\author{
Diane Neff, Sylwia Smuczynska, Jack Simons* \\ Chemistry Department and Henry Eyring Center for Theoretical Chemistry, University of Utah, Salt Lake City, UT 84112, United States
}

\section{A R T I C L E I N F O}

Article history:

Received 5 January 2009

Received in revised form 17 February 2009

Accepted 21 February 2009

Available online 9 March 2009

\section{Keywords:}

Electron transfer dissociation

Electron capture dissociation

Electron transfer

Rydberg states

\section{Introduction and background}

Electron capture dissociation [1] (ECD) and electron transfer dissociation [2] (ETD) mass spectroscopic methods have shown much utility and promise for sequencing peptides and proteins. A strongpoint of both techniques is their propensity for selectively cleaving disulfide and $\mathrm{N}-\mathrm{C}_{\alpha}$ bonds and for doing so over a wide range of the backbone, thus producing many different fragment ions, unlike collision-induced dissociation (CID) or infrared multi-photon dissociation (IRMPD). ECD and ETD also preserve labile side-chains with post-translational modifications. Parallel with many advances in the experimental development and improvement of these methods, theoretical studies have been carried out by several groups in several nations to try to determine the mechanism(s) [3] by which electron attachment leads to these specific bond cleavages as well as how the initial electron attachment occurs.

\footnotetext{
* Corresponding author. Tel.: +1 8015818023.

E-mail address: simons@chem.utah.edu (J.Simons).

URL: http://simons.hec.utah.edu (J. Simons).
}

\subsection{The Coulomb stabilization model}

At an early stage of our efforts [ $3 \mathrm{~h}-3 \mathrm{~m}, 3 \mathrm{o}-3 \mathrm{t}$ ] to explore how ETD or ECD electrons can cleave $\mathrm{S}-\mathrm{S}$ or $\mathrm{N}-\mathrm{C}_{\alpha}$ bonds in positively charged gas-phase peptides, we proposed that electrons can attach directly (i.e., in a nearly vertical exothermic process) to $S-S \sigma^{*}$ or OCN amide $\pi^{*}$ orbitals, but only under special conditions. In particular, we suggested that such low-lying empty orbitals can have their energies lowered by attractive Coulomb interactions with positively charged groups (e.g., the protonated amine or fixed-charge groups on side) thus rendering the electron attachment exothermic. We first put forth this model in 2003 to explain SS cleavage [3j]; we $[3 \mathrm{~h}-3 \mathrm{~m}, 3 \mathrm{o}-3 \mathrm{t}]$ and the Turecek group [3a-3f] later extended the model to electron attachment to amide $\pi^{*}$ orbitals relating to $\mathrm{N}-\mathrm{C}_{\alpha}$ bond cleavage. In Scheme 1, we illustrate the mechanisms by which such electron attachment events have been proposed to lead to cleavage of disulfide or $\mathrm{N}-\mathrm{C}_{\alpha}$ bonds through what we termed Coulomb-stabilized direct electron attachment and what is sometimes called the UW (Utah-Washington) mechanism.

In the $\mathrm{N}-\mathrm{C}_{\alpha}$ cleavage case, two possible structures for the c-type fragments are shown; they have identical mass-to-charge ratios, so the mass spectrometry experiment cannot distinguish them. The amide structure (left) is thermodynamically more stable, but, 
depending whether a proton is transferred from elsewhere in the polypeptide before or after the $\mathrm{N}-\mathrm{C}_{\alpha}$ bond cleaves, one would expect either the enol-imine (right) or the amide (left) to be formed, respectively. Recently, a very nice infrared multi-photon action spectroscopic probe [4] of the c-type fragments formed in ECD was carried out and it was determined that the amide structure is formed, not the enol-imine. This provides evidence suggesting that the proton transfer takes place after the $\mathrm{N}-\mathrm{C}_{\alpha}$ bond has cleaved, not before [5].

What caused us to suggest this Coulomb stabilization model was others' work on dissociative electron attachment [6] showing that, in the absence of Coulomb stabilization, vertical electron attachment to an $\mathrm{S}-\mathrm{S} \sigma^{*}$ or amide $\pi^{*}$ orbital is ca. $1 \mathrm{eV}$ and ca. $2.5 \mathrm{eV}$ endothermic, respectively. So, we knew that very low-energy free electrons (as in ECD) would not be likely to vertically attach; this, of course, suggests it would be even less feasible for ETD electrons to attach. Knowing that the Coulomb potential varies with distance $R$ as $14.4 \mathrm{eV} \AA / R$ with $R$ in $\AA$, we postulated that a disulfide linkage must experience Coulomb stabilization exceeding $1 \mathrm{eV}$ for ECD (even more for ETD because the electron binding energy of the donor anion must also be overcome) to render our directattachment mechanism feasible; this stabilization could arise, for example, from a single positively charged site closer than ca. $14 \AA$, from two positive sites each 28 Å distant, or from a doubly charged site $28 \AA$ away. In a multiply charged polypeptide, all of the Coulomb potentials contribute to this stabilization. Analogously, we postu-

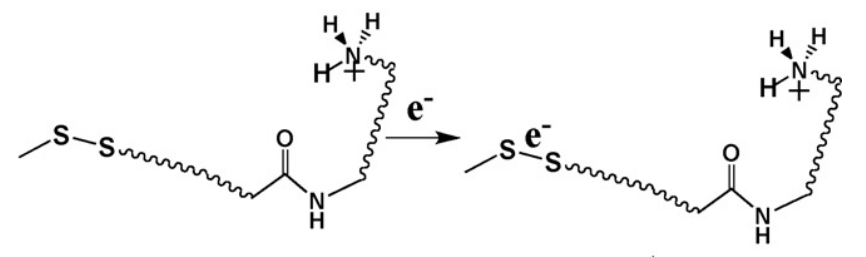<smiles>[H][Y9]([H])([H])[W]O[AsH2]SC</smiles>

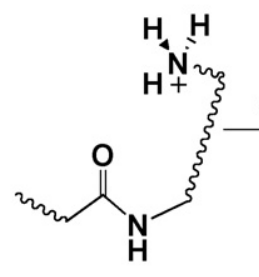

I<smiles>CCCC(CC)NCC</smiles>

II

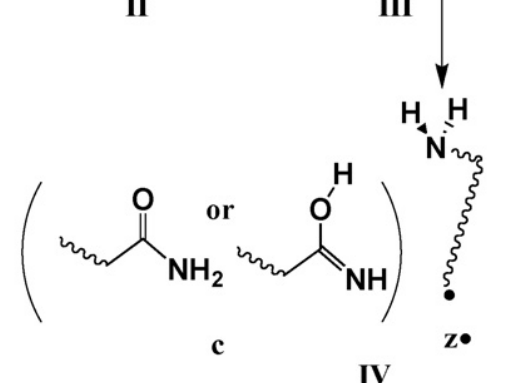

Scheme 1. Direct electron attachment to a Coulomb stabilized S-S $\sigma^{*}$ or OCN $\pi^{*}$ orbital to cleave a disulfide or $\mathrm{N}-\mathrm{C}_{\alpha}$ bond.

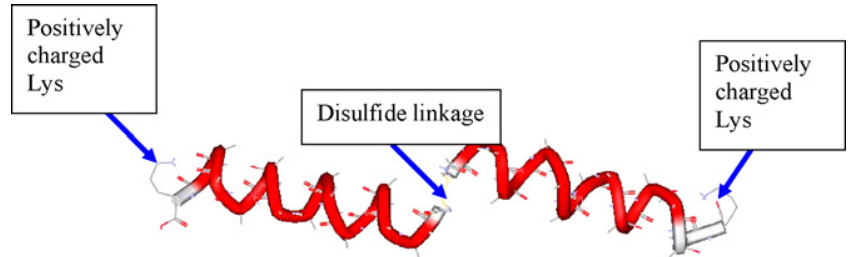

Fig. 1. Assumed structure of doubly charged $\left(\mathrm{AcCA}_{n} \mathrm{~K}+\mathrm{M}\right)_{2}{ }^{2+}$ cations $(\mathrm{M}=\mathrm{H}$ or $\mathrm{Na})$ in gas phase (redrawn from [3j]).

lated that a single positive charge $14.4 / 2.5=6 \AA$ from an OCN $\pi^{*}$ orbital could render this orbital amenable to exothermic direct ECD electron attachment. As we briefly summarize below, these predictions have proven to be consistent with several prior and subsequent experimental observations.

\subsection{Systems of interest in early studies}

The Coulomb-stabilized direct attachment model was used in our first effort [3j] to rationalize the distribution of fragment ions observed [7] in the Marshall lab under ECD conditions for the doubly charged peptides shown in Fig. 1. In this species, two positively charged sites (protonated or sodiated Lys) are held rather far (up to $30 \AA$ A) from a disulfide bond by rigid poly-alanine helices.

The importance of the experimental findings in this case relates to an earlier proposal from the McLafferty lab [1a] that is called the Cornell mechanism. In this mechanism, as applied to the species shown in Fig. 1, an electron is captured at one of the two protonated Lys sites to form a Rydberg species that subsequently undergoes $\mathrm{H}$ atom loss from one of its $-\mathrm{NH}_{3}{ }^{+}$termini. The ejected $\mathrm{H}$ atom then attacks either the $\mathrm{S}-\mathrm{S}$ bond to cleave it (generating an $-\mathrm{S}^{\bullet}$ radical and an $\mathrm{H}-\mathrm{S}$ - unit) or the oxygen of a nearby amide carbonyl group to generate $\mathrm{a}-(\cdot \mathrm{COH})-\mathrm{NH}-$ carbon radical. This carbon radical then undergoes $\mathrm{N}-\mathrm{C}_{\alpha}$ cleavage to generate the enol-imine c-type fragment shown in Scheme 1. The recent observation that ctype fragments are formed as amides rather than enol-imines and the observation [7] that substantial SS bond cleavage occurs for the species shown in Fig. 1 even when 20 alanines are present (and the distance from the putative nascent $\mathrm{H}$ atom and the $\mathrm{SS}$ bond is ca. $30 \AA$ ) was used [3j] to argue against the Cornell mechanism and in favor of the UW mechanism.

In a later effort, we used the same Coulomb stabilization model [3p] to explore the patterns of SS bond cleavage observed in the McLuckey lab for the triply charged compounds shown in Fig. 2. In these compounds, both protonated and fixed-charge sites exist and their distances from the disulfide bond vary and undergo changes due to dynamical motions of the flexible side chains.

For this species, we argued that electron transfer within the charge-reduced polypeptide from a Rydberg orbital on one positive site to such an orbital on another positive site was likely operative. Further details about how the Coulomb-stabilized direct electron attachment mechanism is consistent with what was experimentally observed for these model compounds are given in [3j and $3 \mathrm{p}]$.

\subsection{Questions asked and answers obtained to date}

The primary questions we have addressed on such systems as well as our conclusions are as follows:

1. Is an ECD or ETD electron more likely to attach to a positively charged site (e.g., a protonated amine on a side chain) or to an SS $\sigma^{*}$ or OCN $\pi^{*}$ orbital that has been sufficiently Coulomb stabilized? What are the branching ratios for such attachment events? 

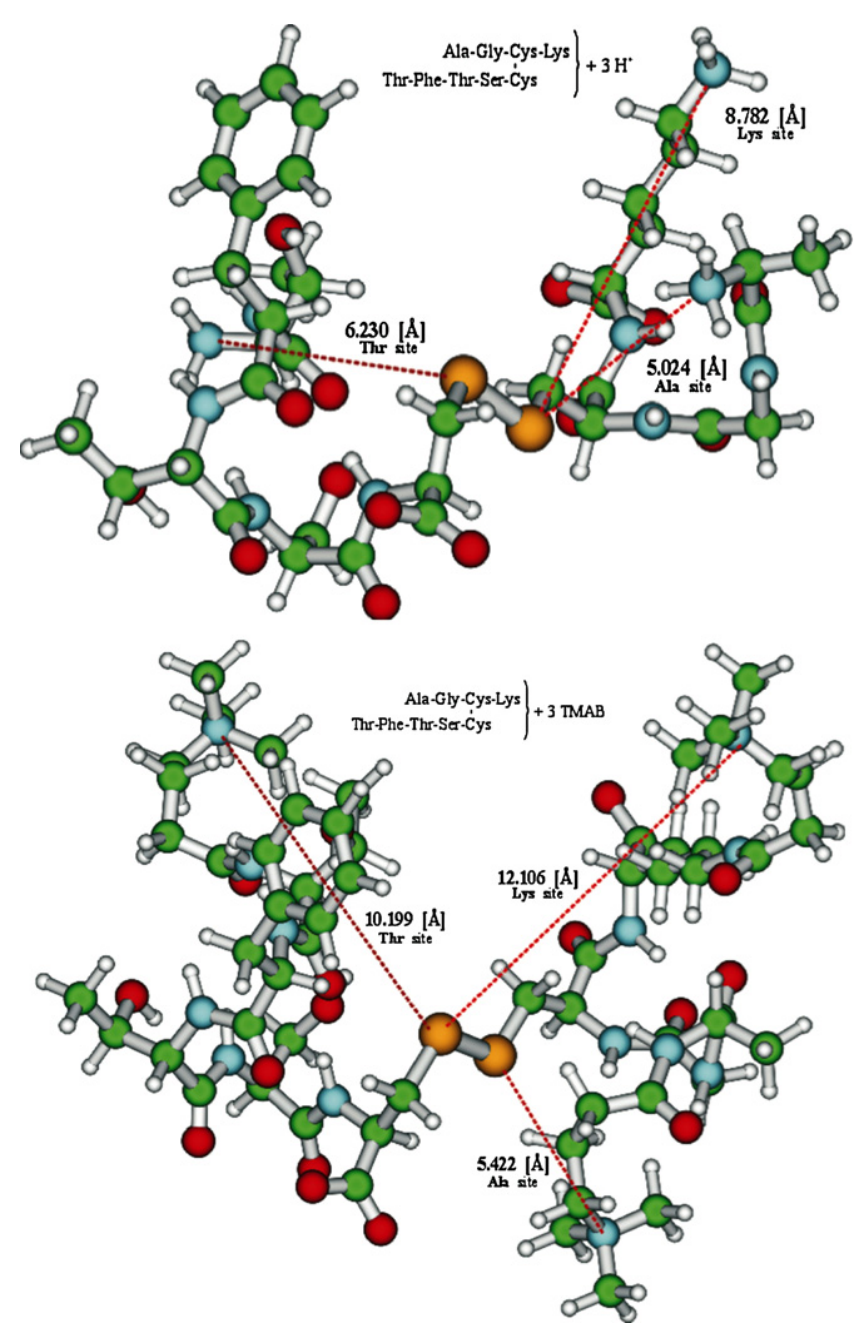

Fig. 2. Triply positively charged ions containing protonated side chains (top) or fixed-charge side chains (bottom) used in the experiments of [2e].

Our Landau-Zener studies [3h,3i], in which we search for potential surface crossings among

a. the entrance-channel ion-pair state in which an ETD anion donor and a positively charged model peptide initiate a collision, and

b. states in which an electron has been transferred from the anion donor either to a 3s or 3p Rydberg orbital of a positive site, to an SS $\sigma^{*}$ orbital, or to an OCN $\pi^{*}$ orbital, showed that the cross-sections for electron transfer to a Rydberg orbital of a positive site are ca. 10-100 times larger than for transfer to an SS $\sigma^{*}$ or an OCN $\pi^{*}$ orbital. So, attachment to a positive site is more likely, but direct attachment to the SS or OCN bond sites can occur.

2. If an electron attaches to an SS $\sigma^{*}$ or OCN $\pi^{*}$ orbital, what then happens?

We found that once an electron enters an SS $\sigma^{*}$ orbital, the SS bond is promptly cleaved [3j,3l]; if it enters an OCN $\pi^{*}$ orbital, a barrier must be overcome [3m] before $\mathrm{N}-\mathrm{C}_{\alpha}$ bond cleavage occurs.

3. If an electron initially attaches to a positive site, can it undergo transfer (through-bond or through-space) to an SS $\sigma^{*}$ or OCN $\pi^{*}$ orbital? If so, at what rates do these processes occur and over what distances are they feasible?

Once an electron attaches to a Rydberg orbital of a positive site, it can undergo through-bond transfer [3p-3s] to an SS $\sigma^{*}$ or OCN $\pi^{*}$ orbital, but only over ca. 6-7 intervening bonds (equivalently, over ca. $15 \AA$ [8]). It can also undergo through-space transfer. The exponential decay of the coupling strengths $H_{1.2}$ relating to the through-bond transfer, which was found for both aliphatic and olefinic linkages, is what so severely limits the range of these electron transfer processes.

4. If an electron initially attaches to a positive site, can it undergo transfer to another positive site? If so, does it matter whether the two positive sites are of similar character (e.g., both protonated amines vs. fixed-charge)?

An electron initially attached to one positive site can undergo transfer to another positive site [3p, 3d, 4s, etc.] but only if the two sites are within ca. $5 \AA$. It does matter whether the two positive sites are of similar character (e.g., both protonated amines vs. one protonated and one fixed-charge); the electron can only transfer if the electron binding strength of the acceptor site is higher than that of the donor.

5. If an electron initially attaches to a positive site, does it matter whether it occupies an excited- or a ground-state Rydberg orbital?

The coupling strengths $H_{1.2}$ connecting ground (3s)- or (lowenergy (3p)) excited-Rydberg states on positive sites to SS $\sigma^{*}$ or OCN $\pi^{*}$ orbitals, both of which decay exponentially with distance, do not differ from one another by more than an order of magnitude [3r]. However, the ground-state Rydberg orbitals on positive sites are known to be subject to prompt (ca. $10^{-12} \mathrm{~s}$ ) dissociation (e.g., a charge-neutralized $-\mathrm{NH}_{3}$ site promptly loses a hydrogen atom) whereas most excited Rydberg states do not so dissociate. We should emphasize that, thus far, we have only been able to study $3 p$ excited Rydberg states, so the conclusions mentioned above should be viewed in that light.

In Fig. 3 we offer a visual representation that summarizes the findings discussed above.

Although much has been learned about how ECD and ETD electron attachment occurs and how attached electrons might migrate throughout the peptide, there is still much to explore. For example, we believe the following issues still need to be clarified:

1. The total cross-sections for ECD and ETD fragment ion yield are large (often 10-100 $\AA^{2}$ ) and seem to scale as the square of the total charge $(Z)$ on the peptide. Does this imply that the initial electron capture event must involve a high-energy Rydberg orbital? Our earlier work showed ETD curve crossings leading

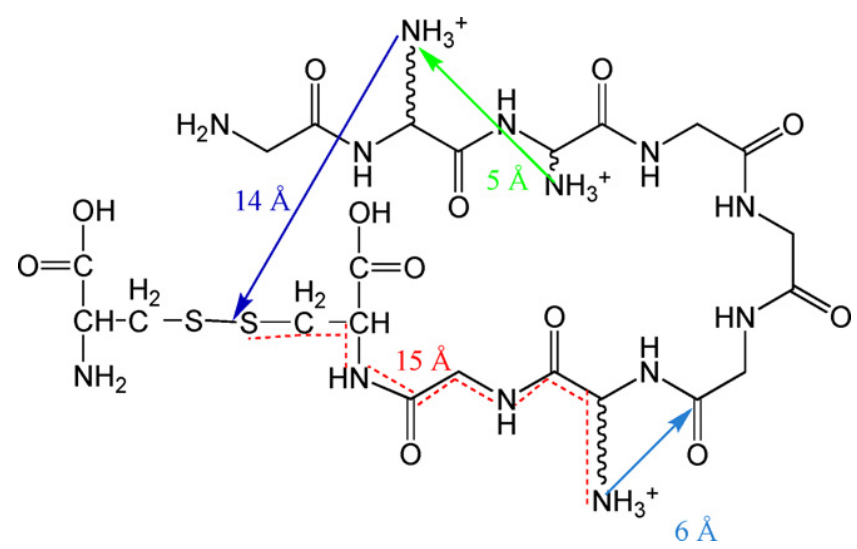

Fig. 3. Representation of the critical distances appropriate to Coulomb-stabilized electron attachment: (dark blue) the $14 \AA$ a single positive charge can be from an SS $\sigma^{*}$ orbital to sufficiently stabilize it; (light blue) the $6 \AA$ the charge can be from an amide $\pi^{*}$ orbital to stabilize it; (red) the $15 \AA ̊$ distance over which through-bond electron transfer can occur; (green) the $5 \AA$ distance over which through-space transfer between positive sites can take place. (For interpretation of the references to color in this figure legend, the reader is referred to the web version of the article.) 
to direct transfer from anion donor to SS $\sigma^{*}$ or OCN $\pi^{*}$ orbitals occur at large distances and can also give large cross-sections (e.g., $10 \AA^{2}$ and larger) if the probabilities of a surface hop are large. Moreover, whenever crossings are involved in the electron attachment event, the cross-sections can be shown to scale as $Z^{2}$, so this scaling law does not necessarily signal the involvement of high-energy Rydberg states. In addition, ETD cannot access as high-energy Rydberg orbitals as can ECD (because the anion donor's electron binding energy must be overcome), yet the fragmentation patterns of ETD and ECD are very similar. Finally, in electron capture-induced dissociation [9] (ECID), where an electron is transferred in a collision with groundstate sodium $\left(3 s^{1} ;{ }^{2} S\right)$ or cesium $\left(6 s^{1} ; 2 S\right)$ atoms and where even greater limitations exist on the energies of the Rydberg states of the peptide can be accessed, the fragmentation patterns are also quite similar. All of these findings seem to suggest that initial attachment to high-energy Rydberg states may not be essential and are consistent with other steps being rate limiting for product-ion yields. In the present work, we show evidence suggesting that excited Rydberg states with principal quantum number $n<10$ are more central to the electron transfer mechanisms.

2. In multiply charged peptides, the SS $\sigma^{*}$ or OCN $\pi^{*}$ orbitals can be very strongly Coulomb stabilized, and the electron binding energies of positive sites' Rydberg orbitals can be enhanced by Coulomb stabilization from the other positive sites. How does the strength of the total Coulomb potential of the peptide alter electron attachment crosssections and through-bond and through-space electron transfer rates (e.g., from positive sites to SS $\sigma^{*}$ or $\mathrm{OCN} \pi^{*}$ sites)?

In the present paper, we carry out simulations on singly and doubly charged model systems in which the distances between positive sites and bond-attachment sites are altered in an attempt to make progress in addressing some of the above issues. Although these simulations relate most directly to ETD, some of our findings relate, as we discuss later, to ECD and ECID as well.

\section{Methods}

First, it is important to explain the strategy we used to construct model compounds on which to carry out ab initio calculations from which we can gain insight into the electron transfer discussed above. For the kind of poly-peptides shown in Figs. 1 and 2 and for most species used in ETD or ECD experiments, the positively charged sites reside primarily on side chains that possess great motional flexibility. This means that, as the peptide undergoes thermal motion in the gas phase, the distances between the positive sites and any SS or OCN group will fluctuate substantially, as will the distances from one positive site to another. As a result, the Coulomb stabilization energy at the $\mathrm{SS}, \mathrm{OCN}$, and positive sites will also fluctuate with time. Ideally then, one would like to model the dynamical motions of the poly-peptide's side chains and backbone and, at each instant of time, compute the rates for electron transfer from an anion donor to SS, OCN, and Rydberg sites as well as the rates of intra-peptide electron transfer. Such an ideal approach is simply not computationally feasible because of the substantial difficulties involved in each electron transfer rate calculation. Therefore, the approach we have undertaken in all of our studies including this one involves:

a. Using small model compounds containing one disulfide or amide unit to limit the computational cost,

b. Fixing the distances between positive sites and SS or OCN bond sites and between positive sites in each calculation (but varying them from one calculation to another) as a way to gain data representative of that particular set of inter-site distances.

This approach allows us to generate a body of data representative of the range of geometries sampled by a poly-peptide undergoing dynamical motions.

In the present studies of electron transfer from the $\mathrm{CH}_{3}{ }^{-}$donor anion to the model compound chosen to represent a disulfide linkage in proximity to a positive site $\mathrm{H}_{3} \mathrm{C}-\mathrm{S}-\mathrm{S}-\mathrm{CH}_{3} \cdots \mathrm{NH}_{4}{ }^{+}$, we first optimized the geometry of the electron accepting moiety $\mathrm{H}_{3} \mathrm{C}-\mathrm{S}-\mathrm{S}-\mathrm{CH}_{3} \cdots \mathrm{NH}_{4}{ }^{+}$at the Hartree-Fock (HF) level with the distance between the nitrogen atom and the nearest sulfur atom held fixed and with the nitrogen and two sulfur atoms remaining collinear throughout the optimization. Then in subsequent calculations, we retained this frozen geometry because (i) we were attempting to model the environment within a peptide or protein in which an $S-S \sigma^{*}$ orbital is Coulomb stabilized by a positively charged site whose location remains fixed and (ii) we wanted to model vertical electron attachment events (this is why we froze the SS distance at its equilibrium value).

In addition, we wanted to extract information about the distance-dependence of the electron transfer rates, so it was important to have the distance from the $\mathrm{S}-\mathrm{S}$ bond to the $\mathrm{NH}_{4}{ }^{+}$site held fixed. We carried out such calculations with

(a) the distance between the ammonium nitrogen and the nearest sulfur atom equal to $8.5 \AA$ A so that the $S S \sigma^{*}$-attached state would have an energy $(1 \mathrm{eV}-14.4 / 8.5=-0.7 \mathrm{eV})$, which is below that of the methyl anion donor $(-0.2 \mathrm{eV})$; this was necessary to for the donation of an electron from $\mathrm{H}_{3} \mathrm{C}^{-}$to the $\mathrm{SS} \sigma^{*}$ orbital to be feasible,

(b) the distance between the ammonium nitrogen and the nearest sulfur atom equal to $14 \AA$, with a second positively charged group [10] located on the opposite side of the MeSSMe along the N-S-S axis $10 \AA$ or $14 \AA$ from its nearest sulfur atom (i.e., with $\mathrm{Li}^{+} \ldots \mathrm{H}_{3} \mathrm{C}-\mathrm{S}-\mathrm{S}-\mathrm{CH}_{3} \cdots \mathrm{NH}_{4}{ }^{+}$as the model electron acceptor); the combination of the two charges' Coulomb potentials stabilized the SS $\sigma^{*}$-attached state to about the same extent [11] as in case (a) but allowed us to explore the role played by the distance between the disulfide bond and the protonated amine group whose Rydberg orbitals can play a role.

Especially for case (b) above, where the ammonium nitrogen and disulfide sulfur atoms are far apart, three sets of s and p extra-diffuse Rydgerg-type basis functions [12] centered on the ammonium ion's nitrogen atom were added to the aug-cc-pVDZ basis sets [13] used for all the other atoms. This kind of basis was shown earlier [12] to be capable of reproducing the energies of several low-energy Rydberg states of nitrogen-centered radicals.

In evaluating the potential energy surfaces pertinent to our studies of electron transfer from $\mathrm{CH}_{3}{ }^{-}$to $\mathrm{H}_{3} \mathrm{C}-\mathrm{S}-\mathrm{S}-\mathrm{CH}_{3} \cdots \mathrm{NH}_{4}{ }^{+}$ and from $\mathrm{CH}_{3}{ }^{-}$to $\mathrm{Li}^{+} \ldots \mathrm{H}_{3} \mathrm{C}-\mathrm{S}-\mathrm{S}-\mathrm{CH}_{3} \cdots \mathrm{NH}_{4}{ }^{+}$, we had to evaluate the energies of the ground- and several excited-Rydberg states as well as the energy of the state in which an electron occupies the Coulomb-stabilized SS $\sigma^{*}$ orbital. To converge HF calculations on several such states and then perform MP2level correlated calculations on each state would, in our opinion, have been impractical. Therefore, we decided to employ the outer valence Greens function (OVGF [14]) option within the Gaussian code to compute the electron attachment energies (for various states) of a system consisting of $\mathrm{H}_{3} \mathrm{C}-\mathrm{S}-\mathrm{S}-\mathrm{CH}_{3} \cdots \mathrm{NH}_{4}{ }^{+}$ or $\mathrm{Li}^{+} \ldots \mathrm{H}_{3} \mathrm{C}-\mathrm{S}-\mathrm{S}-\mathrm{CH}_{3} \cdots \mathrm{NH}_{4}{ }^{+}$, with a neutral $\mathrm{CH}_{3}$ radical having its carbon atom a distance $R$ from the ammonium cation's nitrogen atom. In this way, we obtained the energies of the various electron-attached states in terms of OVGF electron attach- 
ment energies of the parent $\mathrm{H}_{3} \mathrm{C}-\mathrm{S}-\mathrm{S}-\mathrm{CH}_{3} \cdots \mathrm{NH}_{4}{ }^{+} \ldots \mathrm{CH}_{3}$ and $\mathrm{Li}^{+} \ldots \mathrm{H}_{3} \mathrm{C}-\mathrm{S}-\mathrm{S}-\mathrm{CH}_{3} \cdots \mathrm{NH}_{4}{ }^{+} \ldots \mathrm{CH}_{3}$ species. We want to emphasize that we have considerable experience using such Greens function methods because, as early as 1973 [15], our group developed the so-called equations of motion (EOM) for evaluating electron affinities (EAs) and ionization potentials (IPs). Our efforts utilized a Møller-Plesset (MP) approximation to the wave function of the state whose EAs and IPs were to be computed, and our theory provided these energies up through third order in the MP series. The Greens function tools present in the Gaussian program, developed and implemented largely by the Ortiz and Cederbaum groups [14], are closely related to our EOM method although, of course, they have been highly optimized and improved since our early involvement.

The results of such calculations on many electronic states are families of Born-Oppenheimer adiabatic potential energy surfaces. To probe for conditions under which electron transfer should be facile, we search for geometries near which pairs of such curves undergo avoided crossings. In the figures shown later in this paper, some pairs of curves appear to cross. However, in reality, all of the curves we show undergo avoided crossings. If the couplings between a given pair of states are very weak, the "avoidance" may be too small to see at the resolution of the figure, but it is still present. Because we are interested in finding those state couplings that are most likely to produce electron transfer, we focus mainly on pairs of states that display clear and strong couplings as their respective energy surfaces approach and avoid one another.

Finally, we note that all calculations were performed using the Gaussian 03 suite of programs [16], and the three-dimensional plots of the molecular orbitals were generated with the MOLDEN program [17].

\section{Results and discussion}

\subsection{Expectations about where surface crossings can allow electron transfer}

Let us first consider the electronic energy surfaces that pertain to an ETD collision of an electron-donating anion (represented by the alkyl anion in Fig. 4) and a positively charged peptide containing one or more amide units and one or more disulfide linkages as qualitatively illustrated in Fig. 4. In viewing this figure, let us, for example, consider states that can lead to cleavage of the SS bond when the methyl anion donor collides in a manner that brings it in proximity to the SS bond and to the positive site(s) closest to the SS bond.

There are, of course, many additional electron-attached states for such a model peptide that are not displayed in Fig. 4. For example, other positive sites also support manifolds of Rydberg states, and the amide OCN $\pi^{*}$ orbitals may be sufficiently Coulomb stabilized to render their $\pi^{*}$-attached states electronically stable and thus amenable to electron attachment. However, to minimize clutter in the figure, we show only a few of the Rydberg states derived from the positive site closest to the disulfide bond, the SS $\sigma^{*}$ attached state, and the ion-pair state in which the methyl anion holds the excess electron.

The qualitative depictions shown in Fig. 4 can, we believe, also be used to think about the initial electron attachment steps that arise in ECD experiments. In ECD, the free electron can be viewed as being equivalent to an anion donor with zero intrinsic electron binding energy; so, the ion-pair curve would approach zero energy at large- $R$.

On Fig. 4's entrance-channel surface, the anion and positively charged peptide experience a strongly attractive Coulomb potential as illustrated by the descending curve. The $R$-dependence of this state's energy is expected to be approximately of the form

$E_{\text {ion-pair }}(R)=-0.2 \mathrm{eV}-Z 14.4 \mathrm{eV} / R($ in $\AA$ ),

where $0.2 \mathrm{eV}$ is (approximately) the electron binding energy of an alkyl anion [18] such as $\mathrm{H}_{3} \mathrm{C}^{-}$and $Z$ is the total charge of the peptide. We emphasize that the total charge appears in this formula because $-14.4 Z / R$ is the potential experienced by the anion donor at large- $R$.

The SS $\sigma^{*}$-attached and Rydberg states are shown in Fig. 4 to be very weakly dependent on $R$ (except at very small- $R$ where valence repulsions set in, but these distances are not depicted in Fig. 4) because the donor is no longer negatively charged in these states. The SS $\sigma^{*}$-attached state is expected to have an energy of approximately $+1 \mathrm{eV}$ minus the Coulomb stabilization potential $(C)$ at the SS bond site produced by all of the positive charges in the peptide:

$E_{\sigma *}=1 \mathrm{eV}=\Sigma_{\mathrm{J}} Z_{J} 14.4 / R_{J}=1 \mathrm{eV}-C$

where $Z_{J}$ is the charge of the $J$ th charged group and $R_{J}$ is its distance from the SS bond, and, as explained earlier, $1 \mathrm{eV}$ is the energy required to insert an electron vertically into an SS $\sigma^{*}$ orbital.

The 3s ground Rydberg state on the charged site closest to the SS bond will have an energy of approximately-(3-4) eV minus the Coulomb stabilization potential at its $-\mathrm{NH}_{3}{ }^{+}$site produced by all of the other positive charges in the peptide. Since, by assumption, this charged site is the one closest to the SS bond, the distances from it to the other charged sites can reasonably be approximated as the distances $R_{\mathrm{J}}$ from the SS bond to these other charged sites. Under this approximation, the energy of the 3s Rydberg orbital is approximately:

$E_{3 s}=-(3$ to 4$) \mathrm{eV}-\Sigma_{J^{\prime}} Z_{J} 14.4 / R_{J}=-(3$ to 4$) \mathrm{eV}-C+Z_{1} 14.4 / R_{1}$

where the prime on $J$ indicates the sum is over all the other charged sites in the peptide. In the second expression in Eq. (3), $C$ is the same Coulomb stabilization energy as in Eq. (2) and $Z_{1} 14.4 / R_{1}$ is the stabilization energy arising from the positive site closest to the SS bond (for the example at hand, an $-\mathrm{NH}_{3}{ }^{+}$having $Z_{1}=1$ ). We label the ground Rydberg state $3 \mathrm{~s}$ to suggest its relationship to the $3 \mathrm{~s}$ orbital of the $\mathrm{Na}^{+}$cation which is isoelectronic with the ammonium cation. The excited Rydberg states would then be labeled 3p, 3d, 4s, 4p, etc. and will have energies of approximately

$E_{n l}=E_{n l}^{0}-\Sigma_{J^{\prime}} Z_{J} 14.4 / R_{J}=E_{n l}^{0}-C+Z_{1} 14.4 / R_{1}$

where $E_{n l}^{0}$ is the energy of the Rydberg state having principle quantum number $n$ and angular quantum number 1 , and $-C+Z_{1} 14.4 / R_{1}$ has the same meaning as in our description of $E_{3 \mathrm{~s}}$.

It is important to note that each of states discussed above has an energy that is equal to its intrinsic energy (i.e., in the absence of any Coulomb stabilization) minus the Coulomb stabilization that state gains when an electron occupies it. Note also that the SS $\sigma^{*}$ state is Coulomb stabilized by an amount $C$ (see the shift in the energy axis of Fig. 4), whereas all of the Rydberg states on the positive site closest to the SS bond are stabilized by a lesser amount, $C-Z_{1} 14.4 / R_{1}$. The fact that the bond-site and charged-site orbitals are differentially Coulomb stabilized will be shown later to be important.

Also note that the total Coulomb stabilization energy $C$ would, for a peptide of given amino acid sequence, be larger when the total charge state $Z=\Sigma_{J} Z_{J}$ is high than where the charge state is low. Thus, $C$ would be larger for higher $Z$ but is not simply proportional to $Z$ because of the varying internuclear distances $R_{J}$ appearing in Eq. (2) where $C$ is defined.

At various distances $R$ between the anion donor and the peptide, the $\mathrm{SS} \sigma^{*}$ and $-\mathrm{NH}_{3}{ }^{+}$Rydberg electronic states may be crossed [21] by the ion-pair state, and it is near such surface crossings (the circles in Fig. 4) that we anticipate electron transfer to occur. The locations of these crossings can be estimated by equating the energy of the 


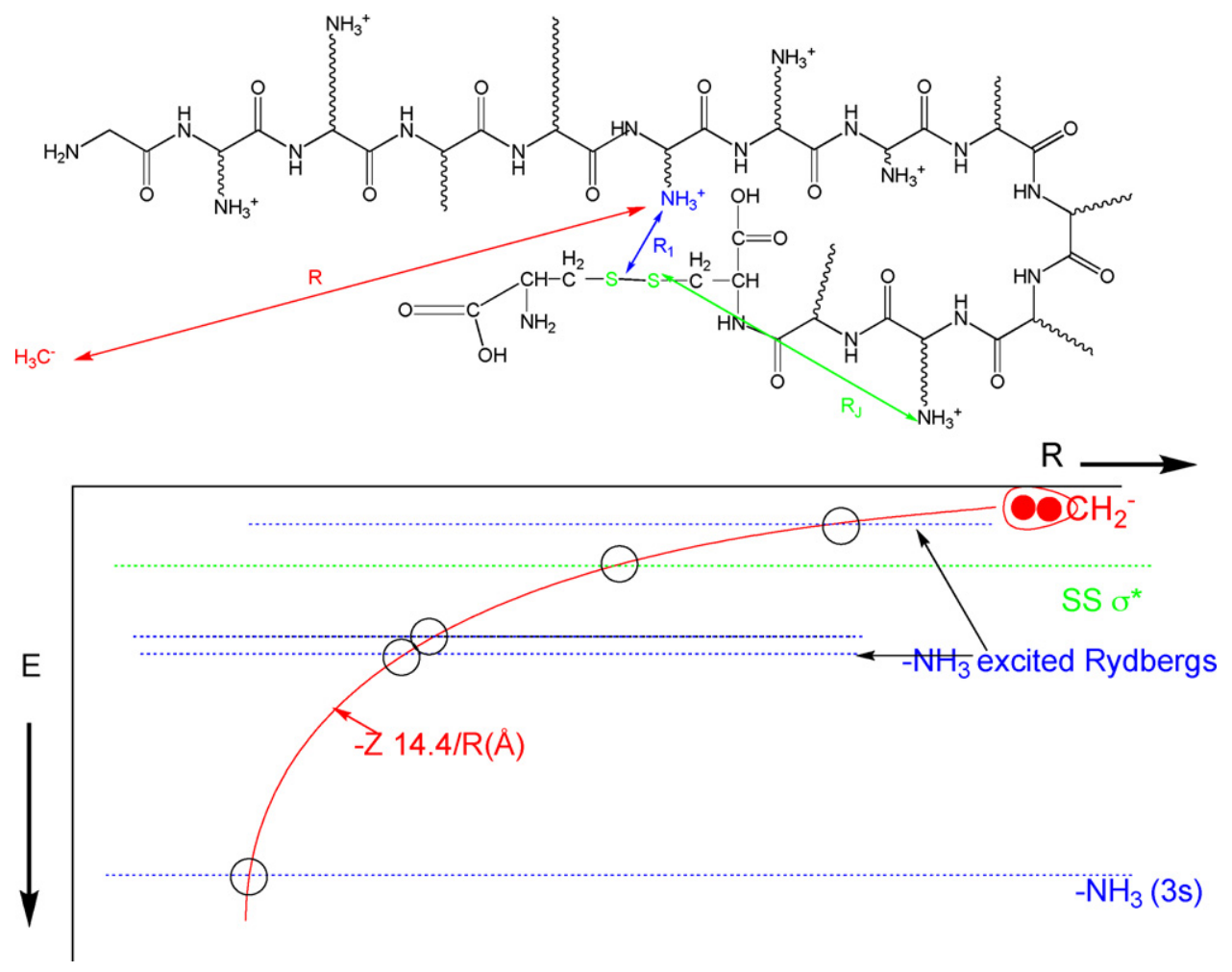

Fig. 4. Qualitative plots of the energies of ion-pair (solid descending line), ground (3s)- and excited-Rydberg (horizontal blue lines), SS $\sigma^{*}$-attached (green line) states as functions of the $\mathrm{CH}_{3}{ }^{-}$donor-peptide cation acceptor separation $(R)$ for the model system shown. (For interpretation of the references to color in this figure legend, the reader is referred to the web version of the article.)

ion-pair state in Eq. (2) with the energy of any of the other states (Eqs. (2)-(4)):

$-0.2 \mathrm{eV}-Z 14.4 \mathrm{eV} / R($ in $\AA$ ) $)=E_{\sigma *, 3 s, n l}$,

which gives

$R_{\text {crossing }}=\left[-0.2-E_{\sigma *, 3 s, n l}\right]^{-1} 14.4 Z$.

To compute cross-sections for electron transfer at such a crossing point, one multiplies the probability $P$ of an electron transfer taking place at the crossing by $\pi R_{\text {crossing }}^{2}$ :

$\sigma=\pi R_{\text {crossing }}^{2} P$.

Because $R_{\text {crossing }}$ is proportional to the total charge $Z$ (Eq. (6)), any cross-section thus evaluated will be proportional to $Z^{2}$. So, the observation of $Z^{2}$ scaling in the ECD or ETD product ion yields does not point to the involvement of high-n Rydberg states.

In earlier studies [3h,3i], we made use of Landau-Zener (LZ) theory to estimate the probabilities and cross-sections for electron transfer from the ion-pair state to each of the other states shown in Fig. 4 as well as to an amide $\pi^{*}$ orbital when studying $\mathrm{N}-\mathrm{C}_{\alpha}$ bond cleavage. In this way, we were able to estimate the branching ratios for electron transfer to ground or low-energy excited Rydberg sites (thus forming a hypervalent $-\mathrm{NH}_{3}$ radical) or to an SS $\sigma^{*}$ or OCN $\pi^{*}$ orbital. This approach is expected to be applicable and reliable when the energy profiles of the ion-pair and other electronic states undergo crossings such as shown in Fig. 4. In particular, the LZ approach requires that

(i) in the region where pairs of energy surfaces cross, their geometry dependences can be well approximated by straight lines with fixed slopes $\mathrm{d} V_{1} / \mathrm{d} R$ and $\mathrm{d} V_{2} / \mathrm{d} R$, (ii) (ii) in a crossing region, the coupling between the two states can be characterized by an off-diagonal Hamiltonian matrix element $H_{1.2}$ that is geometry independent, and

(iii) only two energy states be involved in coupling at any particular crossing point.

We should note that we have been able to include only lowenergy excited Rydberg states in our studies thus far because of the great difficulty in describing such states and their couplings within the atomic orbital basis sets employed in conventional quantum chemistry method such as we employ. This means that we are not able to address events involving attachment (ECD or ETD) of an electron into a high-energy Rydberg orbital, although the present study allows us to move to considering higher Rydberg states.

With the expectations illustrated in Fig. 4 in mind, let us now see what was actually found in the calculations we carried out.

\subsection{Actual results}

The first model species we chose was $\mathrm{H}_{3} \mathrm{C}-\mathrm{SS}-\mathrm{CH}_{3} \cdots \mathrm{NH}_{4}{ }^{+}-$ dimethyl disulfide with an ammonium cation at a fixed distance 8.5 $\AA$ from the nearest sulfur atom [19]. This choice for the internal geometry of our model was made because the Coulomb stabilization energy $(14.4 \mathrm{eV} \AA / 8.5 \AA=1.7 \mathrm{eV})$ is enough to render the anion formed by attaching an electron to the SS $\sigma^{*}$ orbital stable (by ca. $0.7 \mathrm{eV}$ ) with respect to electron loss. The second model species we chose was $\mathrm{Li}^{+} \ldots \mathrm{H}_{3} \mathrm{C}-\mathrm{SS}-\mathrm{CH}_{3} \cdots \mathrm{NH}_{4}{ }^{+}$-dimethyl disulfide with an ammonium cation at a fixed distance of $14 \AA$ from the nearest sulfur atom and with a second positive site [10] 10 or $14 \AA$ from the other sulfur atom. The anion we chose to employ was $\mathrm{CH}_{3}{ }^{-}$; this choice allowed us to keep the electronic structure calculations less time consuming, to avoid issues of steric hindrance, and was a choice we had made in earlier studies [3h,3i] to which we could thus compare. Moreover, the electron binding energy of this anion is small 

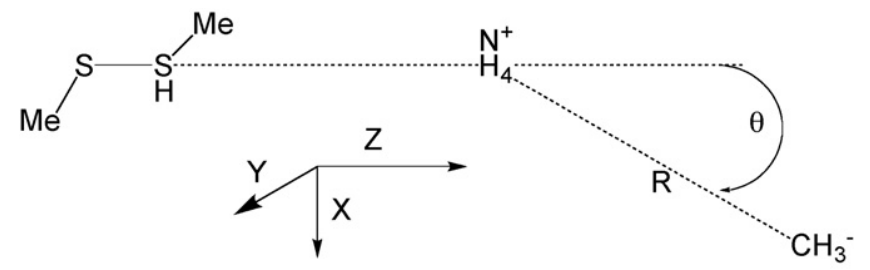

Scheme 2. Definition of coordinates $(R, \theta)$ used to define the location of the anion donor relative to the MeSSMe $\ldots \mathrm{NH}_{4}^{+}$unit as well as the Cartesian directions used to define the $\mathrm{p}_{\mathrm{x}}, \mathrm{p}_{\mathrm{y}}$, and $\mathrm{p}_{\mathrm{z}}$ orbitals.

enough to allow electron transfer to occur to an SS $\sigma^{*}$ orbital that is Coulomb stabilized by $1.7 \mathrm{eV}$.

We examined the energies of the states mentioned above as functions of the distance $R$ between the methyl anion's carbon atom and the ammonium cation's nitrogen atom for several values of the angle $\theta$ between the model compound's S-S- $\mathrm{CH}_{3} \ldots \mathrm{NH}_{4}{ }^{+}$ sulphur-nitrogen internuclear axis and the methyl anion's carbon atom (see Scheme 2). By considering angles of $180^{\circ}, 135^{\circ}, 90^{\circ}$, and $45^{\circ}$, we hoped to gain information that would allow us to consider what happens in collisions with a variety of inter-ion orientations. In our earlier studies, we had only considered collisions near $180^{\circ}$. Clearly, at other angles, the methyl anion's electron donating orbital would be expected to have varying degrees of overlap, and thus varying couplings, with the SS $\sigma^{*}$ and ammonium Rydberg orbitals.

In Fig. 5, we show energy profiles of the first model system (for $\theta=180^{\circ}, 135^{\circ}, 90^{\circ}$, and $45^{\circ}$ ) for states that, at large- $R$, can be characterized by where the electron to be transferred resides:

1. The curve shown in diamonds with energy $0.0 \mathrm{eV}$ at large- $R$ describes the species $\mathrm{H}_{3} \mathrm{C}-\mathrm{SS}-\mathrm{CH}_{3} \cdots \mathrm{NH}_{4}^{+}$plus a neutral $\mathrm{CH}_{3}$ radical (i.e., the species with the electron absent). We show this curve to give the reader some idea of at what geometry repulsion between the $\mathrm{H}_{3} \mathrm{C}-\mathrm{SS}-\mathrm{CH}_{3} \cdots \mathrm{NH}_{4}{ }^{+}$and $\mathrm{CH}_{3}$ sets in (near $2 \AA$ ). We also show it because this is the parent system to which we add an electron in our Greens function [14] calculations to generate the various states of immediate interest, and its energy at large- $R$ is what we use as our reference point of zero energy for all graphs.

2. The curve shown in filled squares having the lowest energy at large- $R$ describes the species $\mathrm{H}_{3} \mathrm{C}-\mathrm{SS}-\mathrm{CH}_{3} \cdots \mathrm{NH}_{4}$ with the electron in the ground-Rydberg (3s) orbital of the $\mathrm{NH}_{4}$, plus a $\mathrm{CH}_{3}$ radical. As expected, its energy lies ca. 3-4 eV below (depending on the Rydberg basis set used) that of the parent system discussed above, reflecting the ground-state electron binding energy of the $\mathrm{NH}_{4} 3 \mathrm{~s}$ orbital within our level of calculation. It does not display any Coulomb stabilization because there are no other positively charged sites in this model system.

3. The curve shown as rapidly descending at large- $R$ relates to the $\mathrm{H}_{3} \mathrm{C}-\mathrm{SS}-\mathrm{CH}_{3} \cdots \mathrm{NH}_{4}{ }^{+}$plus $\mathrm{H}_{3} \mathrm{C}^{-}$ion-pair state. Its energy at large$R$ reflects the electron binding energy of the alkyl radical [20].

4. The relatively flat curve having an energy at large- $R$ near $-0.7 \mathrm{eV}$ relates to the state in which an electron occupies the SS $\sigma^{*}$ orbital. Its electron binding energy $(0.7 \mathrm{eV})$ derives from the Coulomb stabilization $(C)$ of the ammonium cation $8.5 \AA$ away $C=14.4 / 8.5=1.7 \mathrm{eV}$ minus the intrinsic endothermicity (ca. $1 \mathrm{eV}$ ) associated with attaching an electron to an SS $\sigma^{*}$ orbital.

5. The three other curves shown only in the 3-9 $\AA$ region derive from excited 3p Rydberg states of the $\mathrm{NH}_{4}$ system. We do not plot these states' energies beyond $R=9 \AA$ to minimize clutter in the figure, but it is straightforward to visualize how they evolve (linearly) for larger $R$. In this and other figures shown later, the $\mathrm{p}_{\mathrm{y}}$ orbital is directed out of the plane of the figure; the $\mathrm{p}_{\mathrm{x}}$ and $\mathrm{p}_{z}$ orbitals lie in the figure plane.
Some of what is expected based on our discussion surrounding Fig. 4 can be seen in Fig. 5. In particular, we see avoided crossings [21] of the ion-pair state with (i) the SS $\sigma^{*}$ state near $R=13 \AA$ and with (ii) the ground-Rydberg 3s state near $R=4 \AA$. The $3 p$ excitedRydberg states cross [21] the ion-pair states near $R=16 \AA$, but we do not show these crossings in Fig. 5 to avoid clutter. We also note that the coupling matrix element $H_{1.2}$, as estimated by $1 / 2$ the splitting between pairs of states [22] undergoing the avoided crossings, is large for the ion-pair and $3 \mathrm{~s}$ ground-Rydberg states $\left(H_{1.2}=\mathrm{ca}\right.$. $0.25 \mathrm{eV}$ ) but very small $\left(\mathrm{a}\right.$ few $\mathrm{cm}^{-1}$ ) for the ion-pair and SS $\sigma^{*}$ states. It was such large differences in $H_{1.2}$ values that gave rise, in our earlier studies, to the conclusion that cross-sections of electron attachment to ground-Rydberg states should be one to two orders of magnitude larger than for attachment to SS $\sigma^{*}$ states. Finally, we see that the pairs of states undergoing avoided crossings indeed seem to have energies that vary rather linearly with $R$ near the crossings. We saw several other examples of such curve crossings in our earlier studies related to ETD and ECD, so we feel confident in using the LZ method to estimate the surface hopping probabilities in such cases.

\subsection{Surprises}

\subsubsection{Singly charged model comound}

However, we also see in Fig. 5 evidence of couplings that are qualitatively different from the two-state avoided crossing cases just discussed and different from what we observed in our earlier studies. In particular, in the region near 5-6 $\AA$, there appear to be interactions among the SS $\sigma^{*}$, the ion-pair, and the three exicted 3p Rydberg states [23]. The fact that the SS $\sigma^{*}$ and ion-pair states are coupled in the 5-6 A range is surprising given the fact that their diabatic energy curves are degenerate near $13 \AA ̊$ but have very different energies for $R$-values ranging inward from $13 \AA$. In addition, it is surprising that the excited-Rydberg states are involved in interactions within this range of $R$-values because their asymptotic (i.e., large- $R$ ) energies do not suggest that they would cross either the ion-pair or SS $\sigma^{*}$-attached state at these $R$-values (they cross the ion-pair curve near $R=16 \AA$ ). Another interesting feature of the data shown in Fig. 5 in the $R=5-6 \AA$ range is that the couplings do not involve isolated interactions between pairs of states. For example, at $\theta=180^{\circ}$, the ion-pair state couples (over a narrow range of distances) near $6.0 \AA$ with the $3 \mathrm{p}_{z}$ excited Rydberg state, then couples (near $5.5 \AA$ ) with the $3 \mathrm{p}_{\mathrm{x}}$ excited Rydberg sate, and then couples with the SS $\sigma^{*}$ state near $5.0 \AA$. . This complicated network of couplings among the $3 p$ excited Rydberg, SS $\sigma^{*}$ and ion-pair states suggests to us that a straigtforward application of LZ theory to estimate the probabilities of hopping from the ion-pair state to any of the others will not work. However, the fact that couplings occur and that their strengths are substantal, as evidenced in the energies by which the ion-pair curve is shifted from its smooth $R^{-1}$ evolution path (ca. several tenths of an eV; see Fig. 5), suggests that electron transfer from the $\mathrm{H}_{3} \mathrm{C}^{-}$donor to the $3 \mathrm{p}$ excited Rydberg and the SS $\sigma^{*}$ states will occur with significant probability in the $R=4-6 \AA$ region.

So, the data shown in Fig. 5 suggest that electron transfer from the methyl anion donor to the SS $\sigma^{*}$ state can occur, albeit with small probability because of the small $H_{1.2}$ value, near $R=13 \AA$, but can also take place near $R=4-6 \AA$ as the ion-pair, 3 p Rydberg and $S S \sigma^{*}$ states all interact. Moreover, the larger strengths of the state couplings in the latter region suggest that transfer at such geometries is more likely.

To further explore what is causing the complex set of state couplings that arise near $R=4-6 \AA$, we examined, at distances greater than $6 \AA$ and less than $5 \AA$ (so we could better identify the orbitalnature of the states undergoing the coupling) the spatial character of the orbitals occupying the electron that moves from the $\mathrm{H}_{3} \mathrm{C}^{-}$ 

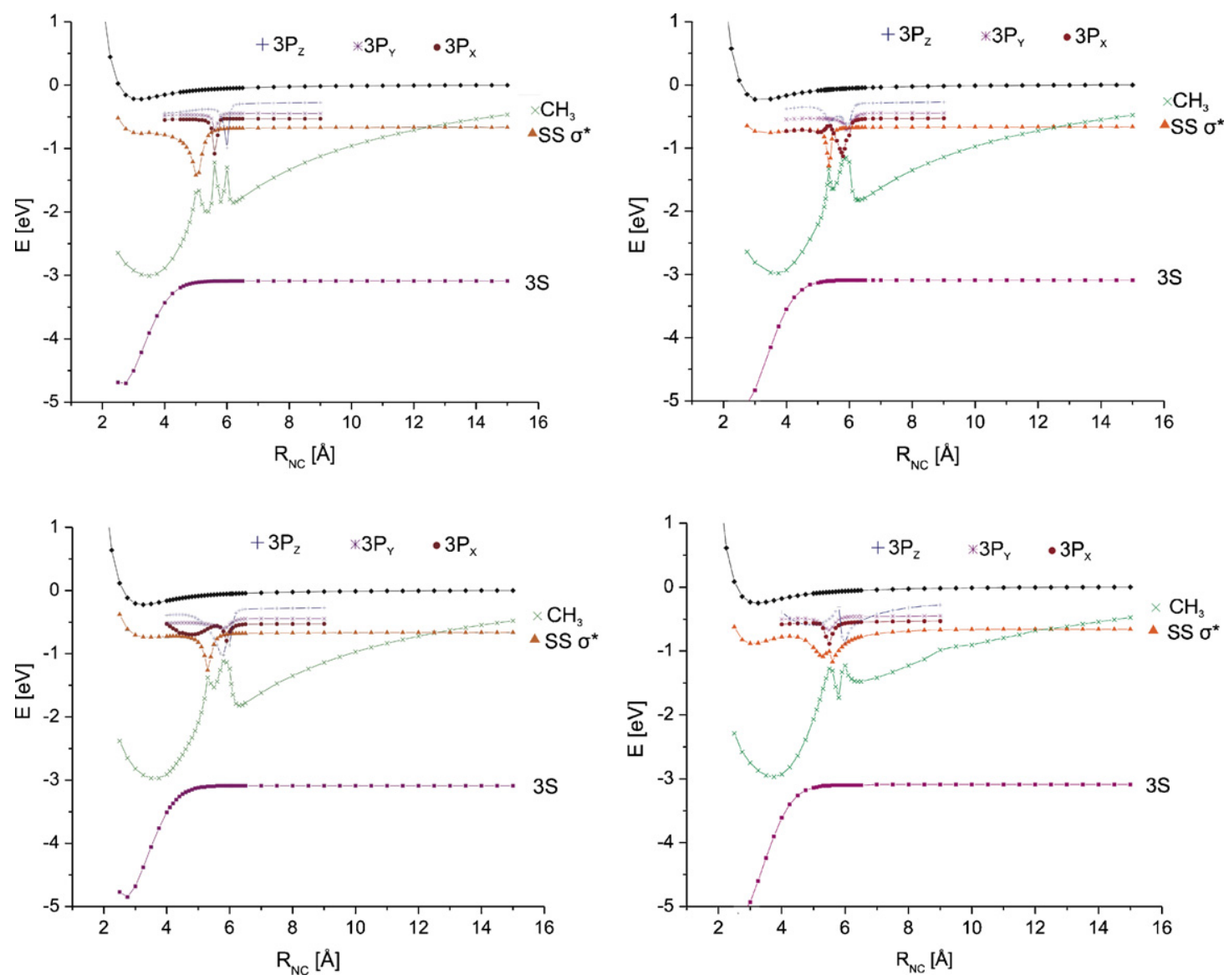

Fig. 5. Energies of ground-Rydberg 3s, SS $\sigma^{*}$-attached, ion-pair, and excited-Rydberg $3 \mathrm{p}$ states as functions of the nitrogen-carbon distance for angles of $180^{\circ}$ (top), $135^{\circ}, 90^{\circ}$, and $45^{\circ}$ (bottom).

anion to the charged peptide. In Fig. 6 we show these orbitals for $\theta=135^{\circ}$ (they do not look qualitatively different at other angles, so this analysis will suffice). The outermost contour for each of the orbitals displayed was selected to guarantee that ca. $80 \%$ of that orbital's electron density lies within that contour. We decided to base our depictions of orbital sizes on this $80 \%$ criterion because experience has shown us that when two orbitals approach one another closely enough for their $80 \%$ contours to "touch", there exists substantial overlap and thus coupling between these two orbitals.

At $R=7.0 \AA$, where we see from Fig. 5 that there is little coupling among the states, the character of each of the orbitals shown in Fig. 6 is straightforward to identify. In Fig. 6a (right column) we see that the ground-Rydberg $3 \mathrm{~s}$ and $\mathrm{CH}_{3}$ lone-pair orbitals are not mixed significantly with any other orbital. The SS $\sigma^{*}$ orbital does seem to experience significant mixing with an ammonium Rydberg orbital. In Fig. $6 b$ (right column) we see that the two 3p excited-Rydberg orbitals directed perpendicular to the $\mathrm{S}-\mathrm{N}$ interatomic axis $\left(3 \mathrm{p}_{\mathrm{x}}\right.$ and $\left.3 p_{y}\right)$ are not significantly coupled to other orbitals. However, the excited-Rydberg orbital directed along the $\mathrm{S}-\mathrm{N}$ axis $\left(3 \mathrm{p}_{z}\right)$ is coupled to the SS $\sigma^{*}$ orbital.

In contrast, at $R=4.5 \AA$ (Fig. 6a left column) there is considerably more coupling among the orbitals. We see that the ammonium $3 \mathrm{~s}$ ground-Rydberg (top left), the $\mathrm{CH}_{3}$ lone pair (bottom left) and SS $\sigma^{*}$ (middle left) orbitals are coupled among one another. Moreover, from Fig. 6b (left column) we see that two of the excited-Rydberg orbitals $\left(3 \mathrm{p}_{z}\right.$ and $\left.3 \mathrm{p}_{\mathrm{x}}\right)$ interact with the $\mathrm{SS} \sigma^{*}$ and $\mathrm{CH}_{3}$ lone pair orbitals; only the $3 \mathrm{p}_{\mathrm{y}}$ excited-Rydberg orbital directed (primarily) out of the plane of the figure remains essentially uncoupled. Looking at the $\theta=135^{\circ}$ energy profiles shown in Fig. 5, we see that the state couplings evidenced by avoided crossings near 5 Å are consistent with these characteristics of the orbitals.

These observations suggest to us that the Rydberg orbitals on the positive sites of an ECD or ETD peptide sample can serve not just as sites to which an electron can be attached (e.g., at the ion-pair ground-Rydberg 3s avoided curve crossing near $R=4 \AA$ or where the $3 p$ excited Rydberg states cross the ion-pair state at $R$-values near $16 \AA$ ) but also as intermediaries that allow an electron to be shuttled from the donor anion to the SS $\sigma^{*}$ orbital in a single step. Specifically, in the case described by data in Fig. 5, we note it is not the ground-Rydberg orbital that is involved in facilitating coupling between the ion-pair and SS $\sigma^{*}$ states probably because the 3s ground-Rydberg state's energy is not close to those of the ionpair and SS $\sigma^{*}$ states. Rather, it is the 3p excited-Rydberg orbitals that have both (i) simultaneous spatial overlap with the methyl lone pair and SS $\sigma^{*}$ orbitals and (ii) energies similar to the energies of the ion-pair and SS $\sigma^{*}$ states, when $R$ is in the 5-6 $\AA$ range.

\section{Doubly charged model comound}

Let us now consider results from a system similar to the MeSSMe $\cdots \mathrm{NH}_{4}{ }^{+}$model but in which the $\mathrm{NH}_{4}{ }^{+}$charged site is farther from the SS linkage and in which a second positive site exists. In particular, we consider a system $\mathrm{Li}^{+} \ldots \mathrm{MeSSMe} \cdot \mathrm{NH}_{4}{ }^{+}$in which the ammonium site is 14 Å rather than 8.5 Å away from the closest sulfur atom and with a second positively charged site $\left(\mathrm{Li}^{+}\right)$located (along the N-S-S internuclear axis) 10 or 14 Å from the closest sulfur atom. The locations of the two positive sites in this model compound are chosen to generate approximately the same Coulomb stabilization 
(a)

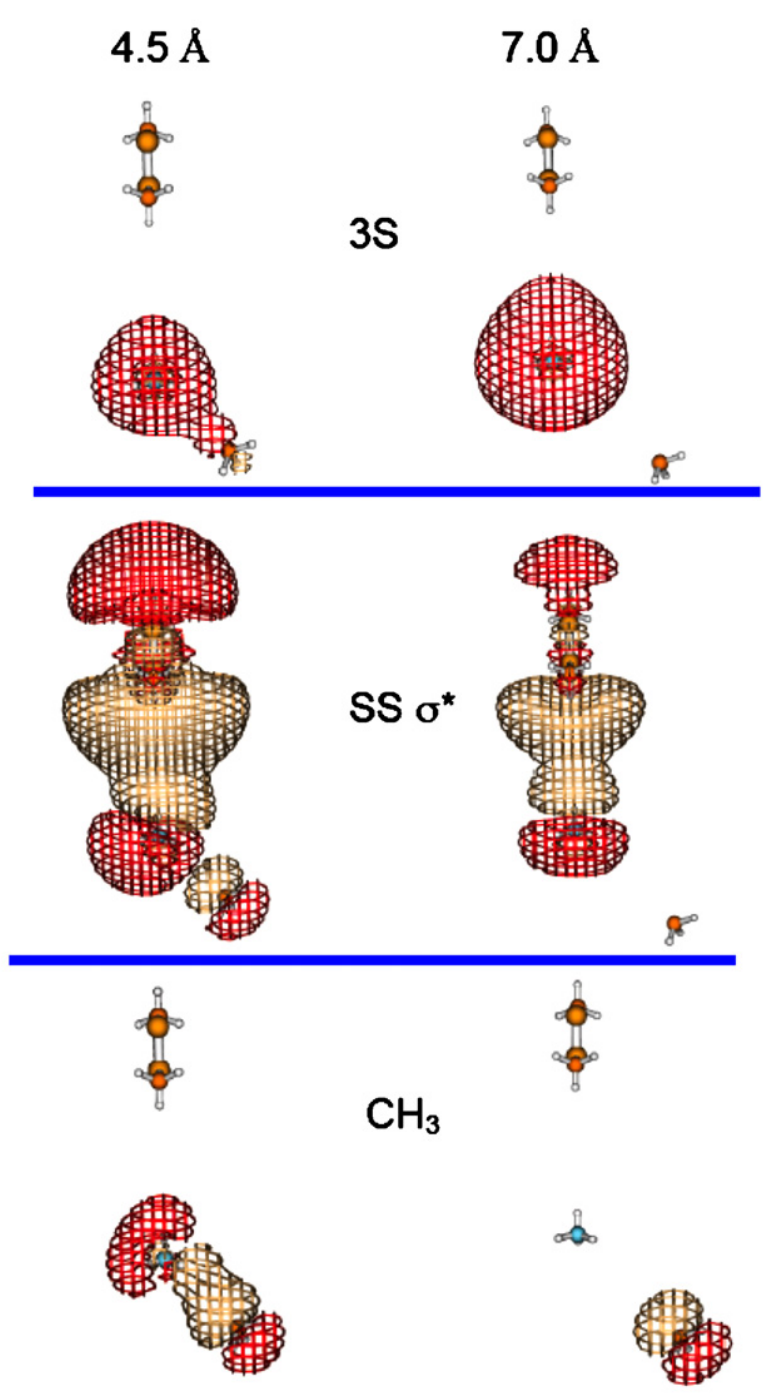

(b) $4.5 \AA$
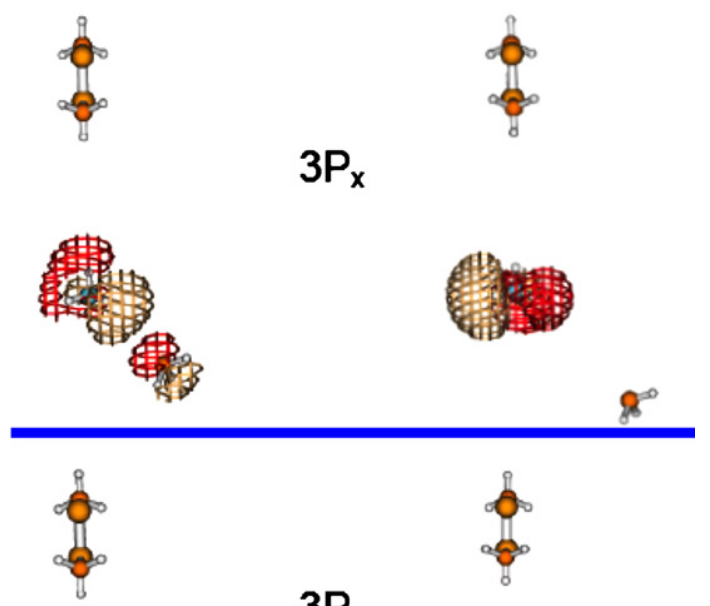

$3 P_{y}$
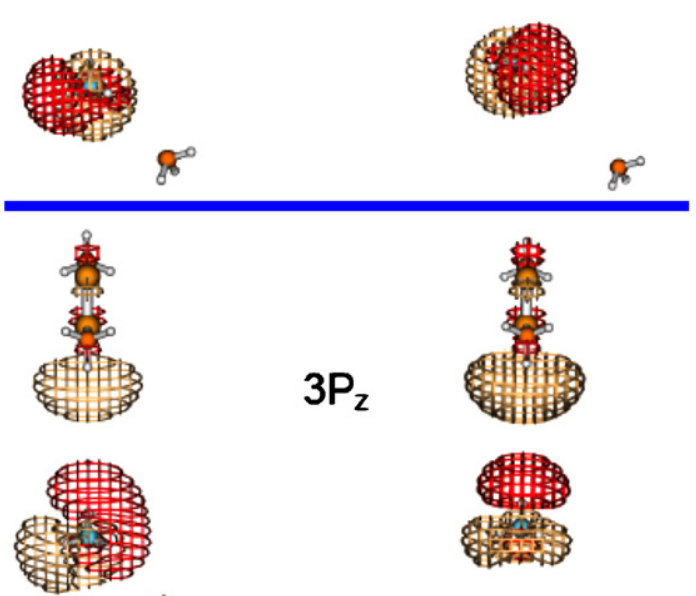

$3 \mathrm{P}_{\mathrm{z}}$
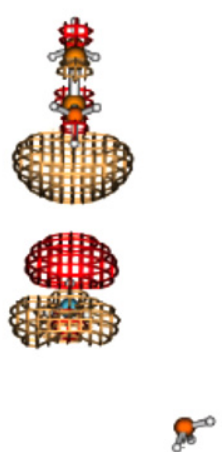

Fig. 6. a. Ground Rydberg (top), SS $\sigma^{*}$ (middle), and $\mathrm{H}_{3} \mathrm{C}$ lone pair (bottom) orbitals at C-N distances of $4.5 \AA$ (left) and $7.0 \AA$ (right), respectively. For each orbital, the $\mathrm{H}_{3} \mathrm{C}-\mathrm{SS}-\mathrm{CH}_{3} \ldots \mathrm{NH}_{4}{ }^{+} \ldots \mathrm{CH}_{3}$ geometry is also shown so one can appreciate the radial sizes of the orbitals involved. b. Three excited Rydberg orbitals: Two localized in the figure plane (top and bottom) and one (middle) perpendicular to the figure plane, at C-N distances of $4.5 \AA$ (left) and $7.0 \AA$ (right), respectively. For each orbital, the $\mathrm{H}_{3} \mathrm{C}-\mathrm{SS}-\mathrm{CH}_{3} \cdots \mathrm{NH}_{4}{ }^{+} \ldots{ }^{-} \mathrm{CH}_{3}$ geometry is also shown so one can appreciate the radial sizes of the orbitals involved.

energy for the SS $\sigma^{*}$ orbital as in the original MeSSMe $\cdots \mathrm{NH}_{4}{ }^{+}$in which the ammonium cation is $8.5 \AA$ from the closest sulfur atom. The choice of placing the $\mathrm{NH}_{4}{ }^{+}$site more distant than in the first model system was made so we could explore which ammonium Rydberg orbitals facilitate shuttling the electron from the anion donor to the SS bond site: Is $14 \AA$ too far for the ammonium 3p orbitals to act as shuttles as they did in the first model compound?

First, it is helpful to recall the sizes and shapes of low-energy Rydberg orbitals such as we study in this work. In Fig. 7, we show 3 s, 3p, 3d, 4s, and 5s Rydberg orbitals of $\mathrm{NH}_{4}$ all drawn to the same scale. In each orbital, the outer surface in the figure contains only $60 \%$ of the electron density (i.e., $40 \%$ of the density lies farther from the cation center). Moreover, for each orbital, one can notice the size of the van der Waals surface of the underlying $\mathrm{NH}_{4}{ }^{+}$cation to gain perspective about how large these Rydberg orbitals are. Realizing that the $\mathrm{N}-\mathrm{H}$ bond length is ca. $1 \AA$, it is easy to appreciate that these Rydberg orbitals span (even at the $60 \%$ contour level) ca. $8 \AA$ for the $3 \mathrm{~s}, 3 \mathrm{p}$, and $3 \mathrm{~d}$ orbitals and even more for the higher-n orbitals [26].

Keeping in mind that the $\mathrm{N}-\mathrm{H}$ bond length in $\mathrm{NH}_{4}{ }^{+}$is ca. $1 \AA$, one can gain some perspective about the radial sizes of these Rydberg orbitals by comparing the size of the underlying $\mathrm{NH}_{4}{ }^{+}$ion to those of the orbitals. The reason the 3s and 3p Rydberg orbitals in Fig. 7 appear smaller than those shown in Fig. 6 is that, in Fig. 6, 80\% of the density resides inside the outermost contour, whereas, in Fig. 7 , only $60 \%$ does. We used the $60 \%$ contour in Fig. 7 so that we could depict the $n=4$ and 5 orbitals within a reasonable space. It should be clear from Figs. 6 and 7 that $n=3$ orbitals (in combination with the radial extent of the SS $\sigma^{*}$ orbital) are large enough to span the $8.5 \AA$ A separating the ammonium and nearest sulfur atom in the first model compound, but may not be adequate to span the $14 \AA$ for the model system currently being discussed (although the SS $\sigma^{*}$ orbital is large enough (see Fig. 6) to assist in spanning these distances).

As stated earlier, we carried out calculations of ion-pair, groundRydberg, excited-Rydberg, and SS $\sigma^{*}$-attached energies for the $\mathrm{Li}^{+} \ldots$ MeSSMe $\ldots \mathrm{NH}_{4}{ }^{+}$doubly charged model system over a range of distances separating the methyl anion donor and the ammonium nitrogen atom. In Fig. 8 we show the energy profiles obtained for an S-N-C angle [24] of $135^{\circ}$ for cases in which the $\mathrm{Li}^{+}$charge is 10 or $14 \AA$ from its nearest sulfur atom. At first glance, the data shown in Fig. 8 is quite similar to those shown in Fig. 5 for the original compound. Near $R=13 \AA$, we see a crossing of the ion-pair and SS $\sigma^{*}$-attached states, and near $R=5 \AA$, an avoided crossing involving 

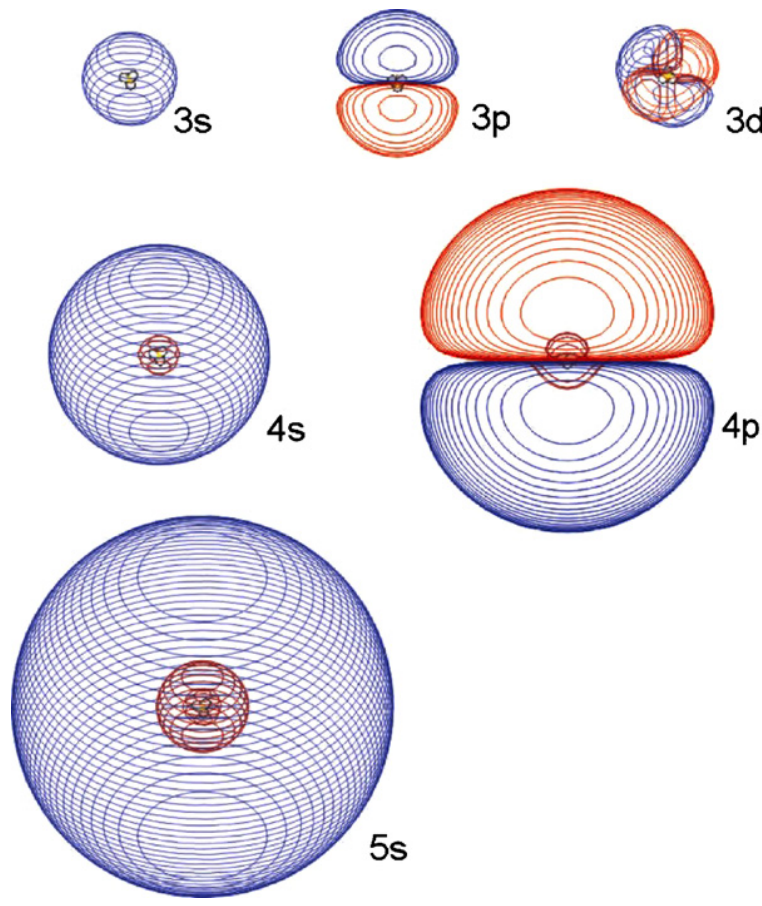

$5 s$

Fig. 7. Plots of 3s, 3p, 3d, 4s, and 5 s Rydberg orbitals of $\mathrm{NH}_{4}$ with the outermost contour containing $60 \%$ of the electron density of that orbital.

the ion-pair and 3s ground-Rydberg states appears. Moreover, at distances somewhat larger than $R=5 \AA$, a complex pattern of state couplings arises, much as with the original compound.

However, there are differences between the findings reported in Figs. 5 and 8 that are important to emphasize:

1. In Fig. 8, the Rydberg states in the doubly charged model compound are all shifted to lower energy than in the singly charged compound. These shifts are due partly [25] to the Coulomb stabilization of all of the ammonium Rydberg states caused by the $\mathrm{Li}^{+}$ion (i.e., recall Eqs. (3) and (4)) but also because of differences in the Rydberg basis sets used in the two studies.

2. In Fig. 8, the SS $\sigma^{*}$-attached state has (by design) approximately the same energy as in the singly charged compound (Fig. 5) when the $\mathrm{Li}^{+}$is $10 \AA$ from its nearest sulfur atom. It has a higher energy when the $\mathrm{Li}^{+}$is $14 \AA$ from the nearest sulfur, also as expected.

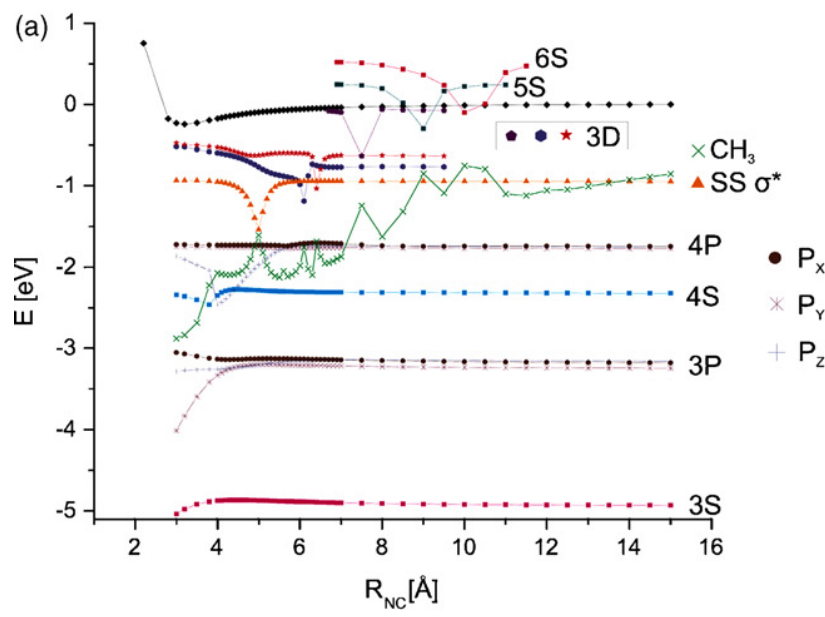

3. In Fig. 8, the SS $\sigma^{*}$-attached state is crossed [21] by the ion-pair state at ca. $R=13-14 \AA$ when the $\mathrm{Li}^{+}$is $10 \AA$ from the nearest sulfur (and thus the SS $\sigma^{*}$ state's energy is similar to that in Fig. 5); this is very near the crossing distance for these two states in Fig. 5.

4. However, in Fig. 8, it is not the 3p Rydberg states that couple with the SS $\sigma^{*}$ state near $R=4-6 \AA$. The $3 \mathrm{~s}$ and $3 p$ Rydberg states have been shifted (by the Coulomb potential of the other charge and by the basis set differences) away from the energy of the $\mathrm{S} \sigma^{*}$ state in this compound. Instead, it is the higher-energy $3 \mathrm{~d}$ Rydberg states that play the "shuttling" role in this case.

5 . The $4 \mathrm{p}, 4 \mathrm{~s}$, and $3 \mathrm{p}$ Rydberg states can be accessed by the methyl anion via avoided crossings near $R=7 \AA$ and inward. However, the 3s Rydberg orbital cannot; there is certainly coupling between the ion-pair and 3s states as evidenced by a strong avioide crossing, but the repulsive part of the potential sets in before this avoided crossing can be accessed.

6 . Because the basis set used in the study whose results appear in Fig. 8 was larger, we also see $3 d, 5$ s, and 6 s Rydberg states that do not appear in Fig. 5. The $5 \mathrm{~s}$ and $6 \mathrm{~s}$ states undergo couplings with the ion-pair state near $R=10$ and $12 \AA$, respectively (i.e., these are the distances at which the methyl anion penetrates the $5 \mathrm{~s}$ and $6 \mathrm{~s}$ orbitals). We do not focus further on these couplings here because the $5 \mathrm{~s}$ and $6 \mathrm{~s}$ states lie (at large $R$ ) above the energy of the ion-pair state and thus cannot be populated in an ETD event.

The most important differences between what is happening in the $\mathrm{Li}^{+} \ldots$ MeSSMe $\cdots \mathrm{NH}_{4}{ }^{+}$and the MeSSMe $\cdots \mathrm{NH}_{4}{ }^{+}$systems are

1. The second positive charge exerts Coulomb stabilization on the manifold of $-\mathrm{NH}_{3}$ Rydberg orbitals, shifting all of these Rydberg orbitals to lower energy relative to the $\sigma^{*}$ orbital. Because the two positive charges' locations were chosen to make the energy of the SS $\sigma^{*}$ orbital (nearly) the same as in the singly charged system, the $-\mathrm{NH}_{3}$ Rydberg states' energies are differentially lowered relative to the energy of the SS $\sigma^{*}$-attached state. This moves the 3 p Rydberg orbitals out of energy-resonance (which they had in the system whose energies are shown in Fig. 5) with the SS $\sigma^{*}$ orbital and moves the 3d Rydberg orbitals into resonance with the SS $\sigma^{*}$ orbital (see Fig. 8).

2. The 3d Rydberg orbitals have enough radial "size" (in combination with the SS $\sigma^{*}$ orbital) to span the $14 \AA$ separating the SS bond and the ammonium's nitrogen atom. As a result, once the methyl anion donor's orbital comes into overlap with the $3 \mathrm{~d}$ Rydberg orbitals, an electron shuttle event through the 3d Rydberg orbitals into the SS $\sigma^{*}$ orbital can occur as evidenced by the complicated set of state couplings in the $R=4-6 \AA \AA$ range.

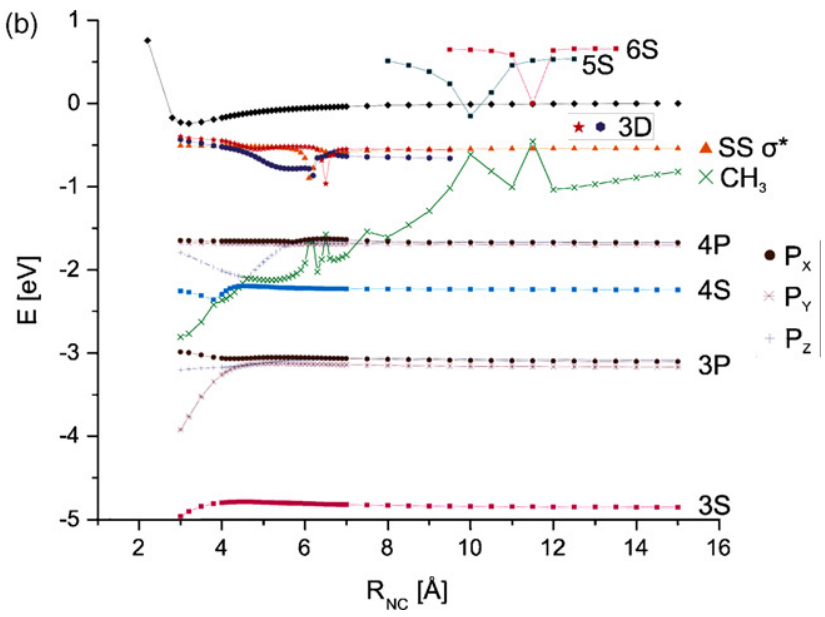

Fig. 8. Energies of ground-Rydberg 3s, $\mathrm{SS} \sigma^{*}$-attached, ion-pair, and excited-Rydberg $3 \mathrm{p}, 4 \mathrm{~s}, 4 \mathrm{p}, 3 \mathrm{~d}, 5 \mathrm{~s}$, and $6 \mathrm{~s}$ states of $\mathrm{Li}^{+} \ldots \mathrm{MeSSMe} \ldots \mathrm{NH}_{4}{ }^{+} \ldots{ }^{-} \mathrm{CH}_{3}$ as functions of the nitrogen-carbon distance, for an angle of $135^{\circ}$, with the nitrogen-sulfur distance at $14 \AA$, and for $\mathrm{Li}^{+}$-sulfur distances of $10 \AA \AA$ (top) and $14 \AA$ (bottom). 
So, the physical picture that arises from this analysis is that through-space (n.b., in our model systems no bonds connect the ammonium and disulfide sites) electron shuttling can occur if:

a. one or more Rydberg orbitals of the positive site nearest the disulfide linkage have an energy (including any Coulomb stabilization effects from other positive charges) that is close to that of the Coulomb-stabilized SS $\sigma^{*}$ orbital, and

b. at least one of those Rydberg orbitals have sufficient radial extent to span the space between the ammonium ion and the SS $\sigma^{*}$ orbital.

Although the particular geometries (i.e., distances from positive sites to the SS bond and between positive sites) and specific basis sets cause specific Rydberg orbitals to play the key shuttling role in a given case, we wish to emphasize that:

a. For a given multiply charged poly-peptide as shown in Fig. 4 containing an SS bond, at any instant in time, the energy of the SS $\sigma^{*}$ orbital will be stabilized by an amount $C$ (Eq. (2)) that depends on the distances from the SS bond to the positive sites in the molecule.

b. At the same instant of time, each positive site's manifold of Rydberg orbitals will have energies (Eqs. (3) and (4)) that depend on the distances to the other positive sites.

c. If a Rydberg orbital on any positive site has both (i) an energy close to that of the SS $\sigma^{*}$ orbital and (ii) sufficient radial extent to couple with the SS $\sigma^{*}$ orbital, then this Rydberg orbital can serve as a shuttle. If the ETD anion donor strikes this orbital when it is in this relationship to the SS $\sigma^{*}$ orbital, electron shuttling can occur.

\section{Summary, conclusions, and overview}

\subsection{Earlier work}

In our earlier works, we considered mechanisms in which

(i) an electron is captured directly into an SS $\sigma^{*}$ or amide $\pi^{*}$ orbital (by the ETD donor anion's participating orbital overlapping the $\sigma^{*}$ or $\pi^{*}$ orbital); for the model compounds being studied here, this event would occur near the curve crossing at $R=13 \AA$ in Fig. 5 or at $R=14 \AA$ in Fig. 8 ,

(ii) or an electron is captured into a Rydberg orbital of a positive site after which, in a separate step, the electron migrates (throughbond or through-space) to the SS $\sigma^{*}$ or amide $\pi^{*}$ orbital.

These prior findings suggested that

(i) because the $H_{1.2}$ values arising in the avoided crossing of the ion-pair and SS $\sigma^{*}$ or amide $\pi^{*}$ states are much smaller than the $H_{1.2}$ values connecting the ion-pair and ground (3s) or lowlying (3p) Rydberg states, only 1 in 100 to 1 in 10 electron capture events occur directly into SS $\sigma^{*}$ or amide $\pi^{*}$ orbitals; most attachment occurs at the positive sites, and

(ii) subsequent to capture at a positive site, the electron can migrate through-bond ca. $15 \AA ̊$ or through-space to an SS $\sigma^{*}$ or amide $\pi^{*}$ orbital. However, we emphasize that these earlier studies only considered transfer from 3 s ground- and 3p excited-Rydberg states; in the present study, we include more excited-Rydberg states (see Fig. 8).

\subsection{Current findings}

However, the results of the present work suggest it is possible that excited Rydberg orbitals on the positive site nearest an SS bond (or, by extension, nearest an amide site) can also play a qualita- tively different role. Specifically, they suggest that excited Rydberg orbitals,

(i) whose energies (n.b., their Coulomb stabilization by the other positive charges in the peptide must be considered) are near the Coulomb-stabilized energy of the SS $\sigma^{*}$-attached state (or amide $\pi^{*}$ when $\mathrm{N}-\mathrm{C}_{\alpha}$ bond cleavage is considered) and

(ii) that have sufficient radial extent to overlap the SS $\sigma^{*}$ (or amide $\pi^{*}$ ) orbital, can shuttle an electron from the anion donor to an SS $\sigma^{*}$ (and probably to an amide $\pi^{*}$ ) orbital through the intervening Rydberg orbital(s).

Because the shuttling mechanism's couplings among several electronic states (SS $\sigma^{*}$, Rydberg, and ion-pair) occur at modest donor-peptide distances (e.g., $R=4-6 \AA$ ) but have substantial interaction strength, this shuttling mechanism is expected to have large fragment-ion yield cross-sections.

In our first model system, the excited 3p Rydberg states, whose energies are shown in Fig. 5 and whose orbitals are depicted in Fig. 6, lie close in energy to the SS $\sigma^{*}$ state over a wide range of distances, including near $R=13 \AA$ 为 where the ion-pair state is also close in energy. However, at such large $R$-values, the methyl lone pair orbital has very little overlap with these excited Rydberg orbitals, so these 3p Rydberg orbitals cannot help shuttle an electron from the methyl anion to the SS $\sigma^{*}$ orbital at such large distances. However, once the methyl anion approaches $R=6 \AA$, it is close enough to the ammonium ion for its lone-pair orbital to overlap these $3 p$ excited Rydberg orbitals which (see Fig. 6) are already in spatial contact with the SS $\sigma^{*}$ orbital (and have energies close to the SS $\sigma^{*}$ orbital). This is when the electron shuttle can occur.

In our second model system, it is the excited 3d Rydberg orbitals that are close in energy to the SS $\sigma^{*}$ state (see Fig. 8). Once the methyl anion approaches $R=6 \AA$, it is close enough to the ammonium ion for its lone-pair orbital to overlap these $3 d$ excited Rydberg orbitals which are already in spatial contact with the SS $\sigma^{*}$ orbital (and have energies close to the SS $\sigma^{*}$ orbital). This is when the electron shuttle can occur.

\subsection{Main conclusions about shuttling}

We therefore suggest that when the ETD donor comes close enough to have substantial overlap with the key excited-Rydberg orbital, it can shuttle an electron through this excited-Rydberg orbital to the SS $\sigma^{*}$ orbital. But, this can occur only if this excitedRydberg orbital is simultaneously in overlap with and close in energy to the SS $\sigma^{*}$ orbital. It is such three-orbital proximities and similarities in energies that give rise to the complex set of avoided crossings seen in Figs. 5 and 8 when $R$ is between 4 and $6 \AA$. For the singly and doubly charged systems studied here, it was the $3 \mathrm{p}$ and $3 \mathrm{~d}$ orbitals, respectively, that met these criteria of spatial and energetic proximity. For other peptides having different charge states, it will likely be other Rydberg orbitals that meet these criteria because the relative energies of the SS $\sigma^{*}$ (or amide $\pi^{*}$ ) and Rydberg orbitals are governed (see Eqs. (1)-(4)) by the Coulomb stabilizations these orbitals experience ( $C$ and $C-Z_{1} 14.4 / R_{1}$, respectively). For example, for the polypeptide shown in Fig. 1, where the two Lys positively charged sites are ca. 25-30 A from the disulfide linkage, the Coulomb stabilization at the SS site is ca. $1 \mathrm{eV}$, just enough to render exothermic electron attachment to the $\sigma^{*}$ orbital. The Coulomb stabilization experienced by either of the Lys sites from the charge of the other site is ca. $14.4 \mathrm{eV} \AA /(50-60 \AA)=0.2-0.3 \mathrm{eV}$, so the manifold of Rydberg states on each Lys site will be Coulomb stabilized by only this small amount. For a Rydberg orbital to meet the criteria specified above for the system in Fig. 1, it would have to have a small electron binding energy (to be close to the energy of the Coulomb-stabilized SS $\sigma^{*}$ orbital) and a radial extent near 
25-30 $\AA$ (to span the space between the Lys and the SS bond). The formula given in ref. [26] suggests that these criteria could be met by an $n=6-7$ Rydberg orbital.

\subsection{How key Rydberg states can act even without shuttling}

We should admit that it is also possible for the excited (3p or 3d in the two examples considered) Rydberg states to be directly populated, through surface hopping from the ion-pair state near $R=15 \AA$ (see Figs. 5 and 8 ) in the initial electron attachment event. It is even possible that these states could be populated by an electron first attaching to a higher-n Rydberg level [26] (n.b., this could occur in either ETD or ECD) after which a cascade of radiationless or radiative relaxations occur. In either case, the excited Rydberg orbital (3p or 3d for our first and second model systems, respectively), being close in energy to the SS $\sigma^{*}$ state and having sufficient radial extent to span the distance between the SS and ammonium sites, could then transfer the electron to the SS $\sigma^{*}$ orbital in a mechanism as we treated earlier in [3q and 3r]. If, as discussed above, the key Rydberg state is close in energy to the SS $\sigma^{*}$-attached state at the equilibrium S-S bond length (as in Figs. 5 and 8), a crossing of the $\sigma^{*}$ and Rydberg states will occur at an accessible geometry and electron transfer may occur.

\subsection{Connections with ECD, ETD, and ECID}

At this stage of our investigation into how and where electrons attach to and cleave bonds in positively charged peptides, the evidence seems to suggest that:

1. Direct ETD or ECD electron attachment to an SS $\sigma^{*}$ or amide $\pi^{*}$ orbital is possible but less likely than attachment to a Rydberg orbital of a positive site.

2. ECD can directly populate highly excited Rydberg states; ETD can populate only Rydberg states having binding energies stronger than that of the anion donor; and ECID can populate only even lower-n Rydberg states. However, the fact that the product ions and relative abundances observed in ECD, ETD, and ECID are usually very similar, suggests that highly excited Rydberg orbitals do not play a key role in the rate-limiting step of bond cleavage [27]. Thus, it is likely that lower (e.g., $3<n<10$ ) Rydberg states (that may be populated through a cascade of relaxation events in ECD or directly in ETD or in ECID) play the key role in transferring the electron to the SS $\sigma^{*}$ or amide $\pi^{*}$ orbital to effect bond cleavage. To further support these assertions, we note that when an electron is transferred [28] from an excited Rydberg state of a Xe atom to a so-called dipole-bound state of acetonitrile (which as an electron binding energy of $0.02 \mathrm{eV}$ ), it is the $n=13$ Rydberg orbital that plays the key role. In the systems studied here, the electron binding energies of the SS $\sigma^{*}$ and amide $\pi^{*}$ orbitals are much larger than $0.02 \mathrm{eV}$, so Rydberg states with $n<13$ are expected to be involved. Using the equation given in [28] relating the electron-acceptor's binding energy and the most efficient (for donation) Rydberg orbital's $n$-value, we obtain $n=7$ for a binding energy of $0.1 \mathrm{eV}$ and $n=4$ for a binding energy of $0.5 \mathrm{eV}$. So, initial attachment to high-n Rydberg states is not likely for ETD and ECID. We think the preponderance of evidence suggests that it may be that the Rydberg states into which an electron is initially attached in ECD, ETD, and ECID are different (i.e., higher-n in ECD), but that a subsequent cascade of relaxation steps eventually leads (in ECD, ETD. and ECID) to a lower-n Rydberg state that plays the key role in governing the product-ion yields.

3. The most important Rydberg states are likely to be those a. residing on a positively charged group near the SS or amide site whose bond is cleaved, b. having energies (including Coulomb stabilization from the other positive sites) close to the energy of the Coulombstabilized SS $\sigma^{*}$ or amide $\pi^{*}$ state, and

c. having sufficient radial extent $[26,27]$ to span the distance between the positive site and the SS or amide site.

4. It appears that Rydberg orbitals fulfilling the conditions noted immediately above can be involved in shuttling an electron from an ETD donor anion to an SS $\sigma^{*}$ or amide $\pi^{*}$ orbital.

5. It is also possible for such a key Rydberg orbital, once populated subsequent to ECD, ETD, or ECID electron attachment and possible relaxation events, to subsequently transfer an electron to an SS $\sigma^{*}$ or amide $\pi^{*}$ orbital via. a surface crossing between, for example, the repulsive SS $\sigma^{*}$-attached state and the excited Rydberg state along the $\mathrm{S}-\mathrm{S}$ bond stretching coordinate (see Fig. 8 in [3s]).

\section{Acknowledgements}

This work has been supported by NSF Grant No. 0806160. Significant computer time provided by the Center for High Performance Computing at the University of Utah is also gratefully acknowledged.

\section{References}

[1] (a) R.A. Zubarev, N.L. Kelleher, F.W. McLafferty, J. Am. Chem. Soc. 120 (1998) 3265 ;

(b) R.A. Zubarev, N.A. Kruger, E.K. Fridriksson, M.A. Lewis, D.M. Horn, B.K. Carpenter, F.W. McLafferty, J. Am. Chem. Soc. 121 (1999) 2857;

(c) R.A. Zubarev, D.M. Horn, E.K. Fridriksson, N.L. Kelleher, N.A. Kruger, M.A. Lewis, B.K. Carpenter, F.W. McLafferty, Anal. Chem. 72 (2000) 563;

(d) R.A. Zubarev, K.F. Haselmann, B. Budnik, F. Kjeldsen, F. Jensen, Eur. J. Mass Spectrom. 8 (2002) 337.

[2] (a) J.E.P. Syka, J.J. Coon, M.J. Schroeder, J. Shabanowitz, D.F. Hunt, Proc. Natl. Acad. Sci. U.S.A. 101 (2004) 9523;

(b) J.J. Coon, J.E.P. Syka, J.C. Schwartz, J. Shabanowitz, D.F. Hunt, Int. J. Mass Spectrom. 236 (2004) 33;

(c) S.J. Pitteri, P.A. Chrisman, S.A. McLuckey, Anal. Chem. 77 (2005) 5662;

(d) H.P. Gunawardena, M. He, P.A. Chrisman, S.J. Pitteri, J.M. Hogan, B.D.M. Hodges, S.A. McLuckey, J. Am. Chem. Soc. 127 (2005) 12627;

(e) H.P. Gunawardena, L. Gorenstein, D.E. Erickson, Y. Xia, S.A. McLuckey, Int. J. Mass. Spectrom. 265 (2007) 130.

[3] (a) E.A. Syrstad, F. Turecek, J. Phys. Chem. A105 (2001) 11144;

(b) F. Turecek, E.A. Syrstad, J. Am. Chem. Soc. 125 (2003) 3353;

(c) F. Turecek, M. Polasek, A. Frank, M. Sadilek, J. Am. Chem. Soc. 122 (2000) 2361;

(d) E.A. Syrstad, D.D. Stephens, F. Turecek, J. Phys. Chem. A107 (2003) 115;

(e) F. Turecek, J. Am. Chem. Soc. 125 (2003) 5954;

(f) E.A. Syrstad, F. Turecek, Am. Soc. Mass. Spectrom. 16 (2005) 208;

(g) E. Uggerud, Int. J. Mass. Spectrom. 234 (2004) 45;

(h) I. Anusiewicz, J. Berdys-Kochanska, J. Simons, J. Phys. Chem. A109 (2005) 5801 ;

(i) I. Anusiewicz, J. Berdys-Kochanska, P. Skurski, J. Simons, J. Phys. Chem. A110 (2006) 1261;

(j) A. Sawicka, P. Skurski, R.R. Hudgins, J. Simons, J. Phys. Chem. B107 (2003) 13505;

(k) M. Sobczyk, P. Skurski, J. Simons, Adv. Quant. Chem. 48 (2005) 239;

(l) A. Sawicka, J. Berdys-Kochaska, P. Skurski, J. Simons, Int. J. Quant. Chem. 102 (2005) 838;

(m) I. Anusiewicz, J. Berdys, M. Sobczyk, A. Sawicka, P. Skurski, J. Simons, J. Phys. Chem. A109 (2005) 250;

(n) V. Bakken, T. Helgaker, E. Uggerud, Eur. J. Mass Spectrom. 10 (2004) 625; (o) P. Skurski, M. Sobczyk, J. Jakowski, J. Simons, Int. J. Mass Spectrom. 265 (2007) 197;

(p) M. Sobczyk, D. Neff, J. Simons, Int. J. Mass Spectrom. 269 (2008) 149;

(q) M. Sobczyk, J. Simons, Int. J. Mass. Spectrom. 253 (2006) 274;

(r) M. Sobczyk, J. Simons, J. Phys. Chem. B 110 (2006) 7519;

(s) D. Neff, M. Sobczyk, J. Simons, Int. J. Mass Spectrom. 276 (2008) 91;

(t) D. Neff, J. Simons, Theoretical study of electron capture dissociation of $\left[\mathrm{Mg}\left(\mathrm{H}_{2} \mathrm{O}\right)_{n}\right]^{2+}$ clusters, Int. J. Mass Spectrom. 277 (2008) 166-174;

(u) F. Turecek, X. Chen, C. Hao, J. Am. Chem. Soc. 130 (2008) 8818;

(v) X. Chen, F. Turecek, J. Am. Chem. Soc. 128 (2006) 12520;

(w) A.I.S. Holm, M.K. Larsen, S. Panja, P. Hvelplund, S. Brøndsted Nielsen, R.D. Leib, W.A. Donald, E.R. Williams, C. Hao, F. Tureček, Int. J. Mass Spectrom. 276 (2008) 116;

(x) J. Chamot-Rooke, C. Malosse, G. Frison, F. Tureček, J. Am. Assoc. Mass Spectrom. 18 (2007) 2146;

(y) Y.M.E. Fung, T.-W.D. Chan, J. Am. Assoc. Mass Spectrom. 16 (2005) 1523; 
(z) H. Konishi, Y. Yokotake, T. Ishibahsia, J. Mass Spectrom. Soc. Jpn. 50 (2002) 229.

[4] G. Frison, G. van der Rest, F. Turecek, T. Besson, J. Lemaire, P. Matre, J. ChamotRooke, J. Am. Chem. Soc. 130 (2008) 14916.

[5] Although this evidence is convincing, it is no conclusive because the enol-imine could be formed and then undergo intramolecular $\mathrm{H}$ atom transfer if sufficien energy is retained to allow it to overcome the barrier needed to effect this transfer.

[6] C. Dezarnaud-Dandine, F. Bournel, M. Troncy, D. Jones, A. Modelli, J. Phys. B: At. Mol. Opt. Phys. 31 (1998) L497;

M. Seydou, A. Modelli, B. Lucas, K. Konate, C. Desfrancois, J.P. Schermann, Eur. Phys. J. D35 (2005) 199.

[7] R. Hudgins, K. Håkansson, J.P. Quinn, C.L. Hendrickson, A.G. Marshall, in: Proceedings of the 50th ASMS Conference on Mass Spectrometry and Allied Topics, Orlando, Florida, June 2-6, 2002, A020420 (Fig. 1 first appears in publication in ref. $3 \mathrm{j})$.

[8] To illustrate how we arrive at this conclusion, we note it was shown in $3 q$ that coupling strengths in the $300 \mathrm{~cm}^{-1}$ range produce surface-hopping probabilities (using Landau-Zener theory) of ca. 0.1-0.5. Thus, we can estimate the rates of through-bond electron transfer by multiplying the $S-S$ vibrational frequency $\nu_{\mathrm{SS}}$ (ca. $1.5 \times 10^{13} \mathrm{~s}^{-1}$ ) by the surface hopping probability $(0.1-0.5)$ and then scaling by the ratio of the square of $\left(H_{1.2} / 300\right)$ : (a) Rate $\approx(1.5-7.5) \times 10^{12}$ $\left(H_{1.2} / 300\right)^{2} \mathrm{~s}^{-1}$. For the through-bond migration to be effective in cleaving the $\mathrm{S}-\mathrm{S}$ bond, it must occur before the Rydberg species from which the electron is transferred can decay by some other mechanism. It is believed that electron attachment (in ECD or ETD) at a positively charged side chain initially occurs into an excited Rydberg orbital after which a decay cascade leads to formation of the ground Rydberg species. It is known that excited Rydberg states belonging to protonated or fixed-charge amine site undergo radiationless relaxation to the ground Rydberg state in a few to several $\mu \mathrm{s}$. Moreover, we know that the excited Rydberg states do not decay by $\mathrm{N}-\mathrm{H}$ or $\mathrm{N}-\mathrm{C}$ bond cleavage, but the ground Rydberg states do (in ca. $10^{-12} \mathrm{~s}$ ). Hence, to be effective in cleaving an $\mathrm{S}-\mathrm{S}$ bond, the through-bond electron transfer must occur within ca. $10^{-6} \mathrm{~s}$ of the time the electron attaches to an excited Rydberg orbital. This fact allows us to estimate the smallest $H_{1.2}$ coupling strength that could produce $S-S$ bond cleavage by solving Eq. (a) for a rate of ca. $10^{6} \mathrm{~s}^{-1}$. This gives an estimate of $H_{1.2}^{\min }=0.11-0.24 \mathrm{~cm}^{-1}$. Using our earlier $H_{1.2}$ data, we concluded that throughbond electron transfer can occur at a rate capable of yielding $\mathrm{S}-\mathrm{S}$ bond cleavage if there are up to 7 bonds (aliphatic or olefinic) or ca. $15 \AA$.

[9] A.I.S. Holm, P. Hvelplund, U. Kadhane, M.K. Larsen, B. Liu, S.B. Nielsen, S. Panja, J.M. Pedersen, T. Skrydstrup, K. Støchkel, E.R. Williams, E.S. Worm, J. Phys. Chem. A 111 (2007) 9641.

[10] We could have used any positively charged site since the Coulomb stabilization it induces at other sites is independent of the nature of the site. We chose to use the $\mathrm{Li}^{+}$cation because (a) its presence added little to the computational cost and (b) it was easy to identify electron-attached states in which the electron is bound to this cation (e.g. we could easily see Li $2 \mathrm{~s}$ and $2 \mathrm{p}$ states) and to not confuse them with the Rydberg-bound and SS $\sigma^{*}$-bound states we were studying.

[11] In case (a) the Coulomb stabilization was $14.4 / 8.5=1.7 \mathrm{eV}$. In this case, we expect the Coulomb stabilization to be $14.4 / 14$ (from the $\mathrm{NH}_{4}{ }^{+}$) $+14.4 /(14$ or 10 ) (from the $\mathrm{Li}^{+}$) or $2 \mathrm{eV}$ to $2.4 \mathrm{eV}$.

[12] (a) M. Gutowski, J. Simons, J. Chem. Phys. 93 (1990) 3874;

(b) P. Skurski, M. Gutowski, J. Simons, Int. J. Quant. Chem. 80 (2000) 1024

[13] R.A. Kendall, T.H. Dunning Jr., R.J. Harrison, J. Chem. Phys. 96 (1992) 6796.

[14] J.V. Ortiz, J. Chem. Phys. 89 (1988) 6348;

L.S. Cederbaum, J. Phys. B8 (1975) 290;

W. von Niessen, J. Schirmer, L.S. Cederbaum, Comp. Phys. Rep. 1 (1984) 57; V.G. Zakrzewski, W. von, Niessen, J. Comp. Chem. 14 (1993) 13;

V.G. Zakrzewski, J.V. Ortiz, Int. J. Quant. Chem. 53 (1995) 583;

J.V. Ortiz, Int. J. Quant. Chem. Symp. 22 (1988) 431;

J.V. Ortiz, Int. J. Quant. Chem. Symp. 23 (1989) 321.

[15] J. Simons, W.D. Smith, J. Chem. Phys. 58 (1973) 4899.

[16] M.J. Frisch, G.W. Trucks, H.B. Schlegel, G.E. Scuseria, M.A. Robb, J.R. Cheeseman, J.A. Montgomery, Jr., T. Vreven, K.N. Kudin, J.C. Burant, J.M. Millam, S.S. Iyengar, J. Tomasi, V. Barone, B. Mennucci, M. Cossi, G. Scalmani, N. Rega, G.A. Petersson, H. Nakatsuji, M. Hada, M. Ehara, K. Toyota, R. Fukuda, J. Hasegawa, M. Ishida, T. Nakajima, Y. Honda, O. Kitao, H. Nakai, M. Klene, X. Li, J.E. Knox, H.P. Hratchian, J.B. Cross, C. Adamo, J. Jaramillo, R. Gomperts, R.E. Stratmann, O. Yazyev, A.J. Austin, R. Cammi, C. Pomelli, J.W. Ochterski, P.Y. Ayala, K. Morokuma, G.A. Voth, P. Salvador, J.J. Dannenberg, V.G. Zakrzewski, S. Dapprich, A.D. Daniels, M.C. Strain, O. Farkas, D.K. Malick, A.D. Rabuck, K. Raghavachari, J.B. Foresman, J.V. Ortiz, Q. Cui, A.G. Baboul, S. Clifford, J. Cioslowski, B.B. Stefanov, G. Liu, A. Liashenko, P. Piskorz, I. Komaromi, R.L. Martin, D.J. Fox, T. Keith, M.A. Al-Laham,
C.Y. Peng, A. Nanayakkara, M. Challacombe, P.M.W. Gill, B. Johnson, W. Chen, M.W. Wong, C. Gonzalez, J.A. Pople, Gaussian, Inc., Wallingford, CT, 2004.

[17] G. Schaftenaar, J.H. Noordik, J. Comput. Aided Mol. Des. 14 (2000) 123.

[18] Alkyl radicals typically have EAs in the range of a few tenths of an eV. Protonated amines typically bind an electron to their 3 ground-Rydberg orbital by ca. 3-4 eV; they bind an electron to their excited-Rydberg orbitals by smaller amounts as suggested by the Rydberg formula $13.6 \mathrm{eVZ}^{2} /(n-\delta)^{2}$.

[19] The internal coordinates of the $\mathrm{H}_{3} \mathrm{C}-\mathrm{SS}-\mathrm{CH}_{3} \cdots \mathrm{NH}_{4}{ }^{+}$unit were held fixed in order (a) to keep the Coulomb stabilization at the SS bond site fixed and (b) to allow us to simulate the vertical transfer of an ETD electron from the alkyl anion to either the SS $\sigma^{*}$ orbital or to an ammonium ground- or excited-Rydberg orbital.

[20] Because of the $R^{-1}$ dependence of this curve it is difficult to display its asymptotic value on these graphs.

[21] As discussed in Section 2, whenever such a pair of electronic states approach one another, they undergo what is called an avoided crossing. In this and other figures, if the coupling between the two states is strong, one can easily see the two states' energies not actually crossing but "avoiding" one another. If the coupling is very weak, the states appear to actually cross. However, in all of the figures shown in this paper, the states undergo avoided crossings even if the coupling is so small that they seem to actually cross when plotted to the resolution we display. So, whenever we say that two surfaces cross we really mean that they undergo an avoided crossing.

[22] We do not show these values in Fig. 5. We calculated them by evaluating the energies of the two states undergoing the avoided crossing at a series of $R_{\mathrm{NC}}$ values much closer spaced than shown in Fig. 5 . The smallest energy spacing discovered within such finely spaced evaluations was taken to be $2 H_{1.2}$.

[23] To avoid complicating these figures even further we have plotted the energies of states connected to the excited-Rydberg states only in this narrow range of $R$-values. At large- $R$ their energies are relatively "flat" (i.e. much like that of the ground-Rydberg state) and converge to energies expected for members of such Rydberg progressions.

[24] From Fig. 5 it can be seen that that qualitative nature of the energy profiles of the various states does not depend much on the S-N-C angle so we decided to display just one set of data here.

[25] As noted in Section 2, we employed a larger atomic orbital basis set on the doubly charged model system primarily to allow us to obtain a larger number of Rydberg states. However this enhanced basis set also lowers the energies we obtained for the $3 \mathrm{~s}$ and $3 \mathrm{p}$ Rydberg states due to enhancements in the variational space.

[26] Hydrogenic and Rydberg orbitals have "sizes" that can be characterized by their expectation values of $r$ and of $r^{2}:\langle r\rangle_{n, l}=\left(n^{2} a_{0} / Z\right)\left[1.5-\left(l(l+1) / 2 n^{2}\right)\right] ;\left\langle r^{2}\right\rangle_{n, l}=$ $\left(n^{4} a_{0}^{2} / Z\right)\left[2.5-\left(3 l(l+1) / 2 n^{2}\right)\right]$ where $n$ and $l$ are the principal and angular momentum quantum numbers of the orbital and $a_{0}$ is the Bohr unit of length $\left(a_{0}=0.529 \AA\right)$. These expressions can be found, for example, in L. Pauling, E.B. Wilson, Jr. Introduction to Quantum Mechanics with Applications to Chemistry, Dover Publications, New York, 1985. To conceptualize the magnitude of the overlap (and thus the $H_{1.2}$ coupling strength) of a Rydberg orbital with, for example, a methyl anion lone pair, an SS $\sigma^{*}$, or an amide $\pi^{*}$ orbital, think of a Rydberg s-orbital as a spherical shell of radius $\langle r\rangle n_{0}=1.5 n^{2} a_{0} / Z$ having a radial "thickness" $\delta r$ to its electron distribution characterized by its dispersion in radial distribution $\delta r=\left[\left\langle r^{2}\right\rangle_{n, 0}-\left(\langle r\rangle_{n, 0}\right)^{2}\right]^{1 / 2}=0.5 n^{2} a_{0} / Z$. This shell of thickness $\delta r$ thus has a surface area of $4 \pi 2.25 n^{4} a_{0}^{2} / Z^{2}$ and a volume of $V_{\mathrm{n}}=4 \pi 2.250 .5 n^{6} a_{0}^{3} / Z^{3}$. In contrast, a methyl anion lone pair, an SS $\sigma^{*}$, or an amide $\pi^{*}$ orbital has a volume of ca. $V_{\text {bond }}=4 / 3 \pi\left(10 a_{0}\right)^{3}$. Now, consider one of the latter orbitals penetrating into a Rydberg orbital, and approximate the electron density within each of the two volumes $V_{\mathrm{n}}$ and $V_{\text {bond }}$ as uniform. That is, within each volume, the respective wave functions are approximated by $\psi(r)=(1 / V) 1 / 2$. The $H_{1.2}$ coupling should then scale with $n$ in the same manner as the overlap integral $(S)$ between the two wave functions $S=\int_{V_{\text {bond }}} 1 / V_{\text {bond }}^{1 / 2} 1 / V_{n}^{1 / 2} d^{3} r=V_{\text {bond }}^{1 / 2} / V_{n}^{1 / 2}=\sqrt{10^{3} Z^{3} / 0.5(3)(2.25) n^{6}}$ given in terms of the square root of the fraction of the volume of the Rydberg orbital terms of the square root of the fraction of the volume of the Rydberg orbital
that is shared with the penetrating orbital of volume $\left(10 a_{0}\right)^{3}$. Even for $n=4$, this overlap is $0.27 Z^{2 / 3}$. For $n=9, S$ is $0.02 Z^{3 / 2}$. This scaling of the overlap between a Rydberg orbital and a valence-sized orbital as $n^{-3}$ suggests that the $H_{1.2}$ couplings will be small except for Rydberg orbitals in the $n=3-10$ range, not for high-n Rydberg orbitals.

[27] It is important to keep in mind that, although a high-n Rydberg orbital has great radial extent, its overlap (and thus its $H_{1.2}$ coupling) with a distant SS $\sigma^{*}$ or amide $\pi^{*}$ orbital will be small as explained in ref. [26].

[28] C. Desfrancois, Phys. Rev. A 51 (1995) 3667. 